\title{
Design, performance, and utilization of the Low-Energy X-ray Reflectometer at DTU space
}

Henriksen, Peter L.; Christensen, Finn E.; Massahi, Sonny; Ferreira, Desiree D.M.; Svendsen, Sara; Gellert, Nis; Jegers, Arne S.; Sokolov, Andrey; Shortt, Brian

Published in:

Journal of Astronomical Telescopes, Instruments, and Systems

Link to article, DOI:

10.1117/1.JATIS.7.4.048004

Publication date:

2021

Document Version

Publisher's PDF, also known as Version of record

Link back to DTU Orbit

Citation (APA):

Henriksen, P. L., Christensen, F. E., Massahi, S., Ferreira, D. D. M., Svendsen, S., Gellert, N., Jegers, A. S., Sokolov, A., \& Shortt, B. (2021). Design, performance, and utilization of the Low-Energy X-ray Reflectometer at DTU space. Journal of Astronomical Telescopes, Instruments, and Systems, 7(4), [048004].

https://doi.org/10.1117/1.JATIS.7.4.048004

\section{General rights}

Copyright and moral rights for the publications made accessible in the public portal are retained by the authors and/or other copyright owners and it is a condition of accessing publications that users recognise and abide by the legal requirements associated with these rights.

- Users may download and print one copy of any publication from the public portal for the purpose of private study or research.

- You may not further distribute the material or use it for any profit-making activity or commercial gain

- You may freely distribute the URL identifying the publication in the public portal 


\title{
Design, performance, and utilization of the Low-Energy X-ray Reflectometer at DTU space
}

\author{
Peter L. Henriksen $\odot$, ${ }^{\text {a, } *}$ Finn E. Christensen, ${ }^{a}$ Sonny Massahi $\odot$, \\ Desiree D. M. Ferreira $\odot,{ }^{\text {a }}$ Sara Svendsen $\odot,{ }^{\text {a }}$ Nis Gellert $\odot,{ }^{a}$ \\ Arne S. Jegers, ${ }^{a}$ Andrey Sokolov $\odot,{ }^{b}$ and Brian Shortt ${ }^{c}$ \\ ${ }^{a}$ Technical University of Denmark, DTU Space, Kgs. Lyngby, Denmark \\ ${ }^{b}$ Helmholtz-Zentrum Berlin für Materialien und Energie, BESSY-II, Berlin, Germany \\ ${ }^{\mathrm{c} E}$ European Space Agency (ESTEC), Noordwijk, The Netherlands
}

\begin{abstract}
A state-of-the-art compact Low-Energy X-ray Reflectometer is in operation at DTU Space and is used to characterize X-ray coatings for the optics of future spaceborne and groundbased telescopes. The reflectometer is housed in a vacuum chamber and operates at $1.487 \mathrm{keV}$, complimenting an existing $8.048 \mathrm{keV}$ reflectometer. With a microfocus source and planeparabolic Kirkpatrick-Baez mirrors, the 0.5 - $\mathrm{mm}$ wide beam is collimated to $<0.75$ arcmin. An actively cooled 2D CCD yields sample alignment precision of $26 \mu \mathrm{m}$ in linear position and 0.18 arcmin in angle. A multilayer monochromator provides a peak reflectance of $43.5 \%$ and beam purity $>99 \%$. The reflectometer has a $2 \theta$ range of 0 deg to $35 \mathrm{deg}$ and dynamic range up to eight orders of magnitude. Techniques are demonstrated to investigate the sample surface morphology and we show the system's capability to detect the presence of atmospheric contaminants on coated mirrors. () 2021 Society of Photo-Optical Instrumentation Engineers (SPIE) [DOI: 10.1117/1 .JATIS.7.4.048004]
\end{abstract}

Keywords: LEXR; $x$-ray optics; $x$-ray reflectometry; thin-film characterization; grazing incidence; reflectometer validation.

Paper 21069 received Jun. 20, 2021; accepted for publication Nov. 1, 2021; published online Nov. 22, 2021.

\section{Introduction}

As part of the testing of $\mathrm{x}$-ray optics and the development of reflective coatings of their mirrors, it is crucial to have characterization capability at reference energies of relevance to the optics in question. A number of future spaceborne and ground-based observatories equipped with x-ray telescopes rely on high throughput of the optics at low energies. ${ }^{1-3}$ To ensure sufficient performance, it is necessary to obtain a detailed understanding of the characteristics of the mirror surfaces, coating structure, presence, and impact of contaminants. ${ }^{4,5} \mathrm{X}$-ray reflectometry (XRR) characterization can be used to qualify deposited coatings, yielding information about film thickness, roughness, film morphology, density, etc. ${ }^{6}$ Single-energy reflectometers are compact and easy to operate, making them ideal for immediate and follow-up characterization of manufactured $\mathrm{x}$-ray mirrors.

To investigate thin-film performance of particularly low-Z materials and the effects of hydrocarbons and other surface contaminants, as well as mirror manufacturing process steps, e.g., ESA's Advanced Telescope for High-Energy Astrophysics (ATHENA) mission on the performance of coatings, ESA has acquired a state-of-the-art Low-Energy X-ray Reflectometer (LEXR) that has been installed and commissioned at DTU Space. LEXR operates at $1.487 \mathrm{keV}$ and in combination with the existing $8.048 \mathrm{keV}$ reflectometer at DTU Space, enables quick inhouse characterization capability at two reference energies. Until recently the majority of the mirror characterization effort for ATHENA has been carried out at energies above $3 \mathrm{keV}$ with results indicating ${ }^{7-10}$ that low-energy characterization is required for complete evaluation of the impact of post-coating processing of mirror samples. Particularly for the case of bilayer and

*Address all correspondence to Peter L. Henriksen, peter@lindquist-henriksen.dk 
multilayer coatings with low-density overcoatings on high-density reflectors. ${ }^{11,12}$ LEXR has thus been commissioned to allow for investigation of mirror coating reflectivity at $1.487 \mathrm{keV}$.

While XRR measurements at $8.048 \mathrm{keV}$ provide useful information on films consisting of multilayer coatings or single layer coatings of a high- $\mathrm{Z}$ material, qualification of a low- $\mathrm{Z}$ overcoat on a dense film gets increasingly difficult with higher energies due to their reduced reflectance and the dominance in the signal of the high-density layer underneath. On the other hand, x-ray reflectivity measurements at low energies are more susceptible to anomalous effects due to, e.g., hydrocarbons as well as attenuation caused by contaminants on the reflective surface. ${ }^{13-15}$ For this reason, it is important to understand the sources of uncertainty on the measurements to determine not only the capabilities of an XRR system but also its limitations.

In this paper, we present the design, performance and utilization of LEXR, the intermediate stages of which has previously been reported in Refs. 16 and 17. We examine the performance of each of the components and demonstrate how sample information can be extracted by performing different types of measurements. Finally, we discuss the uncertainty on measurements and limitations on information we can extract from the system and compare the system to equivalent synchrotron measurements.

\section{Reflectometer Design and Component Performance}

The reflectometer is housed in a cylindrical vacuum chamber to prevent attenuation of the lowenergy photons in air. Its structure has been designed by JJ X-ray in collaboration with DTU Space based on an older system used for soft $x$-ray studies from which the vacuum chamber has been re-purposed. ${ }^{18}$ The pumps ensure the operating pressure of $<9 \times 10^{-6}$ mbar is reached in $6 \mathrm{~h}$. The beam path is just under $2 \mathrm{~m}$, meaning that the photon attenuation factor for the $1.487 \mathrm{keV}$ setup is of $\mathcal{O}\left(10^{-8}\right)$. The x-ray source and two Kirkpatrick-Baez (KB) collimating mirrors are mounted on an exterior flange of the vacuum chamber, and the chamber itself slides over and houses all the other components of the reflectometer. LEXR operates in the horizontal plane. Figure 1 shows the beam path and main reflectometer components, and Fig. 2 shows the relative positions of components in a side-view drawing.

Slits, goniometers, and sample translation stages are motorized and remotely operated. The monochromator is placed on a goniometer with an angular resolution of 3.6" a linear stage enables positioning with $\mu \mathrm{m}$ precision in the beam. The sample stage has linear translation capability along all three axes, as well as a 360-deg rotational capability. The vacuum chamber wall limits the detector stage to $2 \theta=35$ deg relative to the sample incident beam. The rotational axes of the sample and detector stages are co-aligned to produce $\mathrm{a}<18^{\prime \prime}$ shift in reflected beam across the full $\theta-2 \theta$ range. The sample alignment precision is limited by the detector pixel pitch to $26 \mu \mathrm{m}$ in linear position and $11^{\prime \prime}$ in $\theta$. Motor backlash has been measured to shift alignment by $1.1^{\prime \prime}$.

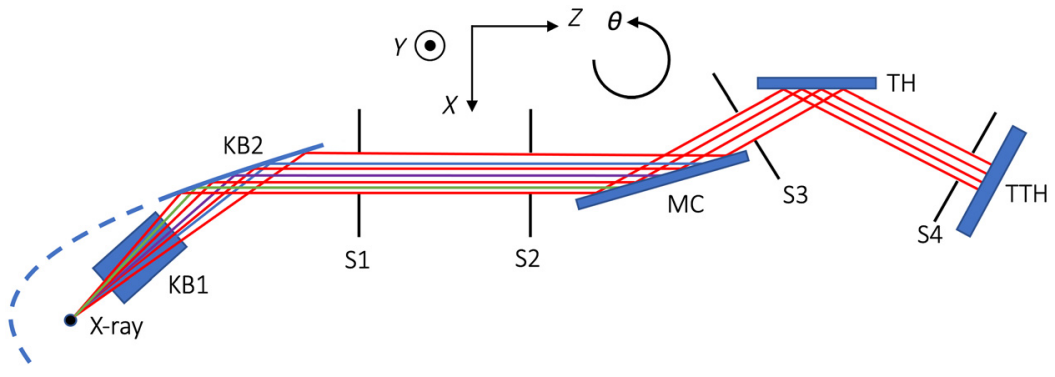

Fig. 1 Top down sketch of LEXR beam path. A: A microfocus source emits a bremsstrahlung spectrum along with characteristic photons. B: Two plane-parabolic KB mirrors collimate the beam in vertical and horizontal plane respectively. C: Slits reduce the level of background. D: A monochromator picks out the $\mathrm{K}_{\alpha}$ line monochromatizing the beam. $\mathrm{E}$ : A slit shaping the beam reaching the sample. F: Sample stage holding the x-ray mirror samples. G: Slit reducing the background level. $\mathrm{H}$ : 2D CCD detector. 


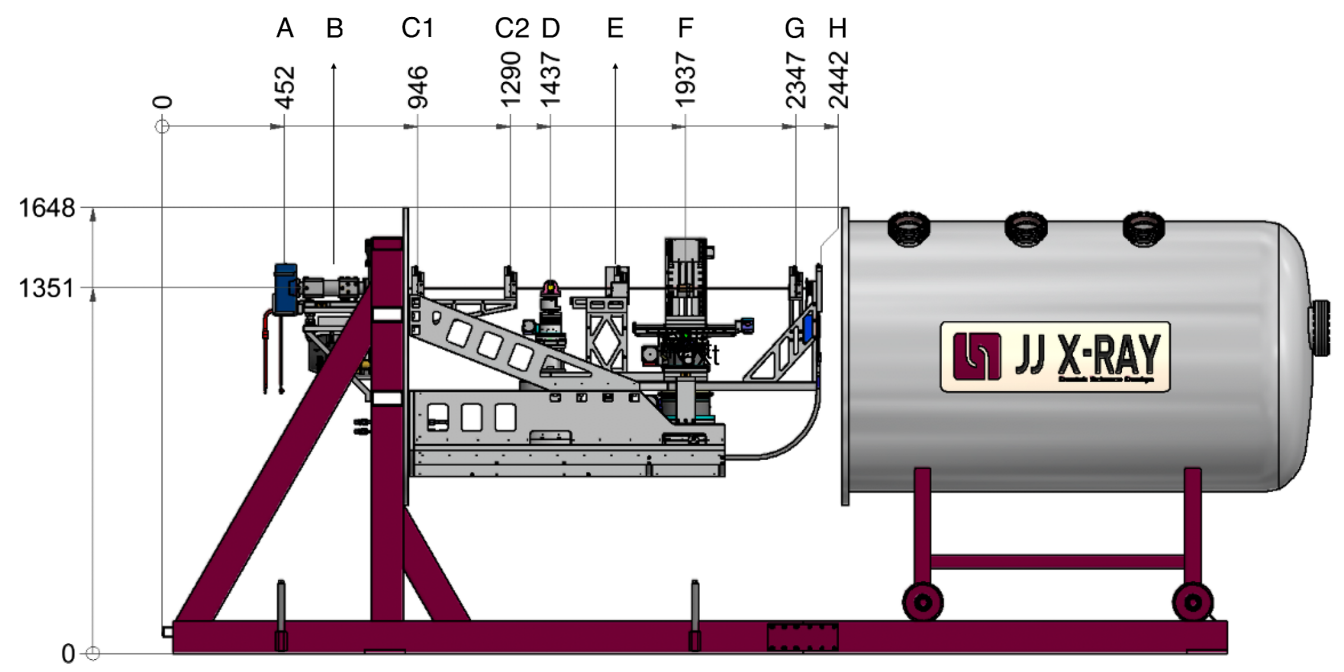

Fig. 2 Side-view drawing of LEXR. Measurements given in mm. A: x-ray source, B: KB mirrors, C: Slits 1 and 2, D: Monochromator, E: Slit 3, F: Sample stage, G: Slit 4, H: Detector.

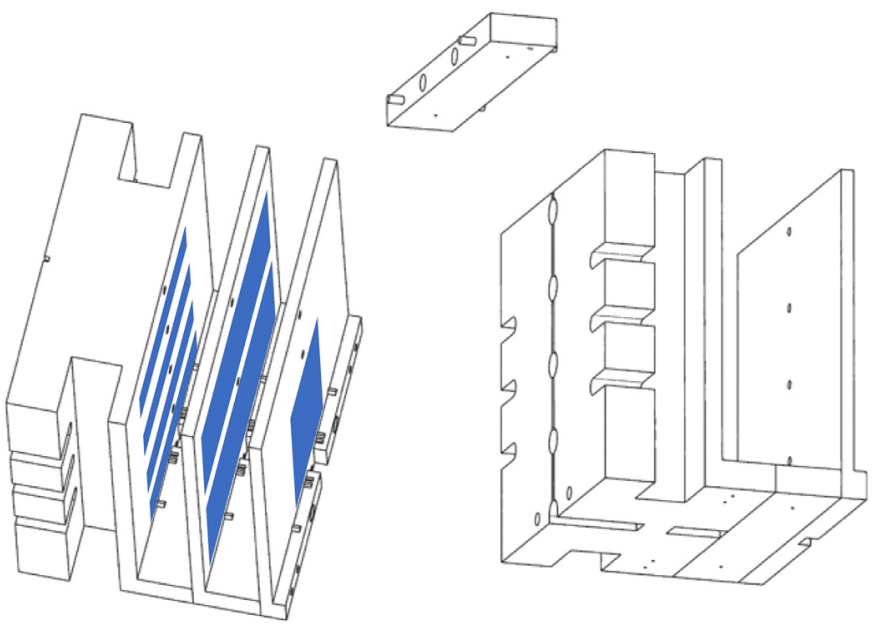

Fig. 3 Isometric drawing of modular sample holder. Left: Standard configuration shown with four samples of $70 \times 10 \mathrm{~mm}^{2}$, two inner radius SPO of $110 \times 49 \mathrm{~mm}^{2}$, and one $40 \times 66 \mathrm{~mm}^{2}$ middle radius SPO. Right: Alternative configuration using distancing piece to increase the sample-tobackplate separation.

To optimize the number of samples that can be measured between each pump-down cycle, and therefore the science output, a modular sample holder is used to enable the mounting of up to twelve mirror samples at once, depending on the sample dimensions (see Fig. 3). The standard configuration of the sample holder has plate-to-plate distance of $21.5 \mathrm{~mm}$, which, for samples of $1-\mathrm{mm}$ thickness and assuming a $0.5-\mathrm{mm}$ beam, yields sufficient sample illumination up to $17.5 \mathrm{deg}$. The sample holder has modularity so the center mounting plate can be replaced with a distancing piece to facilitate mounting of thicker samples or the entire sample holder may be exchanged with one of a different design if needed.

\subsection{X-Ray Source and Mirrors}

LEXR uses a microfocus X-ray source and a pair of plane-parabolic KB mirrors to increase the solid angle of photon collection and to collimate the beam. An Al source with spot size of $50 \times$ $150 \mu \mathrm{m}^{2}$ using $\mathrm{x}$-ray tubes from rtw RöNTGEN-TECHNIK DR. WARRIKHOFF GmbH \& Co. $\mathrm{KG}$ is used in the standard configuration but a $\mathrm{Cu}$ source is available for $8.048 \mathrm{keV}$ measurements. The KB mirrors are $\mathrm{Si}\langle 100\rangle$ substrates produced by Zeiss with rms slope error $<2$ " and 
Table 1 Specifications of mirrors provided by AXO DRESDEN. The multilayer period has a gradient along the KB mirrors, the values stated are nominal at mirror front/center/end respectively.

\begin{tabular}{lccccc}
\hline \hline Mirror & $\begin{array}{c}\text { Source } \\
\text { distance }(\mathrm{mm})\end{array}$ & Multilayer & $\begin{array}{c}\text { Multilayer } \\
\text { period }(\mathrm{nm})\end{array}$ & $\begin{array}{c}\text { of } \\
\text { bilayers }\end{array}$ & $\begin{array}{c}\text { Deflection } \\
\text { angle 2 } \theta \text { (deg) }\end{array}$ \\
\hline $\mathrm{B} 1$ & 130 & $\mathrm{~W} / \mathrm{Si}$ & $2.99 / 4.57 / 5.91$ & 60 & 2.1 \\
$\mathrm{~B} 2$ & 240 & $\mathrm{Ni} / \mathrm{C}$ & $6.15 / 6.49 / 6.83$ & 40 & 1.5 \\
$\mathrm{D}$ & 985 & $\mathrm{~W} / \mathrm{Si}$ & 3.75 & 80 & 13.1 \\
\hline \hline
\end{tabular}

rms surface roughness $<0.2 \mathrm{~nm}$ coated by AXO DRESDEN with laterally graded multilayers optimized for Bragg reflection of $58 \%$ at the $\mathrm{Cu}$ K-edge, whereas the characteristic photons from the $\mathrm{Al}$ source undergo total external reflection. A multilayer coated 1 inch wafer is used as a monochromator to suppress the bremsstrahlung spectrum and $\mathrm{Al} \mathrm{K}$ photons. Details of the mirrors are listed in Table 1.

Two attenuation filters of $100 \mathrm{~nm} \mathrm{Si}_{3} \mathrm{~N}_{4}+100 \mathrm{~nm} \mathrm{Ti}$ are installed in front of the detector to prevent other softer photons from the bremsstrahlung spectrum from entering the detector. The simulations in Fig. 4 show the emission spectrum from the $\mathrm{Al}$ source and the expected resulting spectrum at the detector after interaction with the mirrors and filters. The simulations were carried out by AXO DRESDEN with a $30-\mathrm{eV}$ bin width.

The $\mathrm{Al}$ source is operated at $30 \mathrm{kV}$, which produces a slightly cleaner spectrum due to the large penetration depth of the electrons at this voltage so even though low-energy photons are generated, many are absorbed in the anode material as their generation depth is greater than the mean free path of the photons. Figure 5 shows a $\theta-2 \theta$ measurement of the monochromator around its Bragg angle performed using LEXR. The scan of the monochromator shows peak reflectance of $45 \%$ at $6.53 \mathrm{deg}$, and the best fit gives a W/Si d-spacing of $3.76 \mathrm{~nm}$ with $\Gamma=0.3$ for the 80 bilayers.
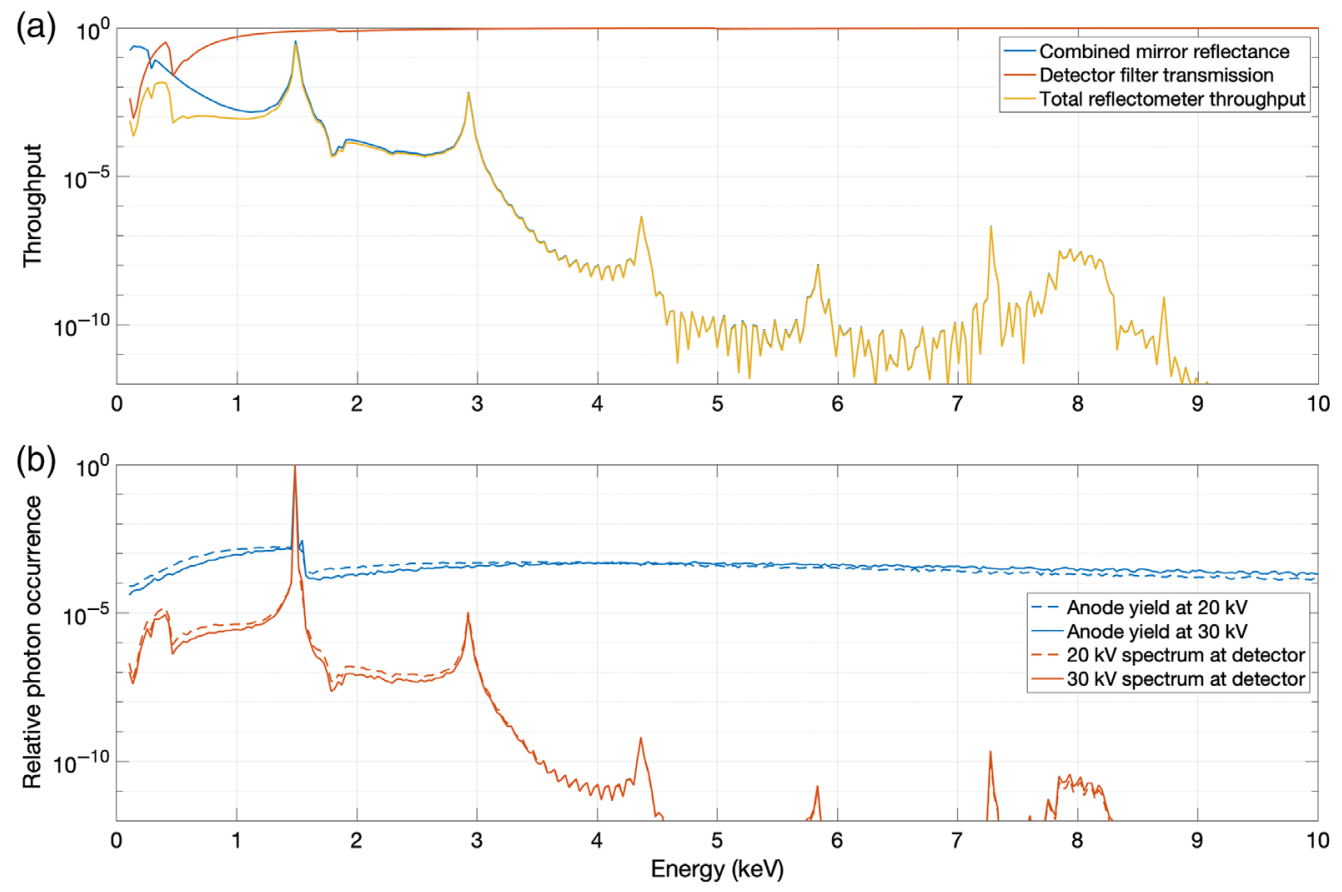

Fig. 4 (a) Simulation of reflectance as function of energy for the center position of the two KB mirrors and the monochromator. Combined with the transmission through attenuation filters, the total throughput to the detector is determined. (b) Simulations of the total anode yield from the Al source running at 20 and $30 \mathrm{kV}$, respectively. With the total component throughput given above, the resultant spectra at the detector are obtained. 


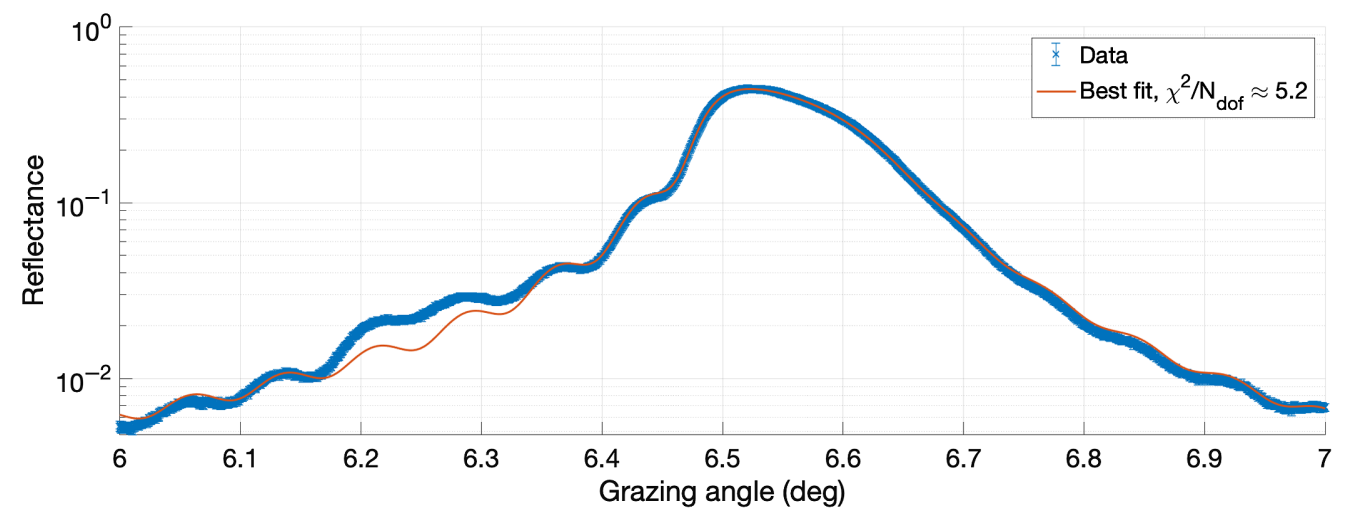

Fig. 5 Reflectivity scan at $1.487 \mathrm{keV}$ scan of the monochromator. The discrepancy between fit and measured data at $6.2 \mathrm{deg}$ to $6.3 \mathrm{deg}$ is due to the Bragg reflection of the $1.55 \mathrm{keV} \mathrm{Al} \mathrm{K}_{\beta}$ photons from this multilayer and is also the cause of the low $\chi^{2}$ probability even with large error bars.

The discrepancy in measured intensity in the region $6.2 \mathrm{deg}$ to $6.3 \mathrm{deg}$ corresponds to the location of the Bragg peak of the monochromator multilayer for the $1.55 \mathrm{keV} \mathrm{Al} \mathrm{K}{ }_{\beta}$ emission line, and the measured signal here is 1.5 times higher than expected for the case of only $\mathrm{Al} \mathrm{K}_{\alpha}$ photons. The measured peak reflectance at $6.54 \mathrm{deg}$ is also slightly higher than expected from the model. This discrepancy matches exactly the ratio of $\mathrm{Al} \mathrm{K} \mathrm{K}_{\beta}$ to $\mathrm{Al} \mathrm{K} \mathrm{K}_{\alpha}$ photons convoluted with the $\mathrm{Al} \mathrm{K} \mathrm{K}_{\beta}$ reflection at this angle. The $\mathrm{Al} \mathrm{K}_{\beta}$ Bragg peak has a reflectance of $43 \%$ of $1.55-\mathrm{keV}$ photons and $15 \%$ of $1.487-\mathrm{keV}$ photons, so the source flux of $1.55-\mathrm{keV}$ photons is around $17.4 \%$ that of $1.487-\mathrm{keV}$ photons. At the $6.54 \mathrm{deg}$ incidence angle of the monochromator, the reflectance of $1.487-\mathrm{keV}$ photons is $43.5 \%$ and that of the $1.55-\mathrm{keV}$ photons is $1.7 \%$. With the ratio of $\mathrm{K}_{\alpha}$ to $\mathrm{K}_{\beta}$ photons measured, this results in a beam purity of $99.3 \%$.

The radiation from the $\mathrm{X}$-ray source is assumed unpolarized so the polarization of the beam impinging on samples comes from the reflection off the KB mirrors in the source housing and the monochromator. The polarization by reflection is a function of the refractive index of the interface and the angle of incidence. The reflection angles off the KB mirrors are small and as the refractive index in the $\mathrm{X}$-ray regime is close to unity, the beam collimated by the $\mathrm{KB}$ mirrors is partially polarized only on the order of $0.1 \%$. The incidence angle on the monochromator is substantially higher at $\theta=6.54 \mathrm{deg}$, which, using the best-fit model to the monochromator scan, yields a partial s-polarization of $2.5 \%$. The detector is not polarization sensitive but sample reflectance is dependent on beam polarization with increasing importance as function of incidence angle. The polarization in LEXR is so small that the effect on measured sample reflectance is less than one percent.

The typical time to complete a specular reflectivity measurement is around $2 \mathrm{~h}$. Figure 6 shows measurements of the pressure in the reflectometer chamber and the long-term beam intensity over the course of $14 \mathrm{~h}$. The relative standard deviation (RSD) of measured intensity (signal + noise) over any $2 \mathrm{~h}$ period varies between $0.42 \%$ and $0.56 \%$ with a mean around $0.47 \%$. At shallow angles where the reflected signal is strong, the variation is mainly caused by flux variation from the beam (described in Sec. 3.2). At large grazing angles, the largest contributor to variation in the measured beam intensity is by far variation in the noise level of the detector. Exposure times here are typically $30 \mathrm{~s}$, and, over such a period, the mean peak-to-valley is around $1.5 \%$ with a maximum of $2.7 \%$.

\subsection{Angular Resolution}

Assuming a flawless KB surface, the horizontal and vertical divergence after the KB mirrors is given by the source size and mirror focal length by $\Delta \theta_{\mathrm{B} 1 \text {,vert }}=(0.15 \times 180 \times 60) /(130 \pi) \approx$ $3.967^{\prime}$ and $\Delta \theta_{\mathrm{B} 2 \text {,hor }}=(0.05 \times 180 \times 60) /(240 \pi) \approx 0.716^{\prime}$. The two slits between the KB mirrors and monochromator do not further reduce beam divergence. From Bragg's law, a relationship between beam divergence and line broadening may be found, here calculated using the fact

J. Astron. Telesc. Instrum. Syst. $\quad 048004-5 \quad$ Oct-Dec 2021 • Vol. 7(4) 
(a)
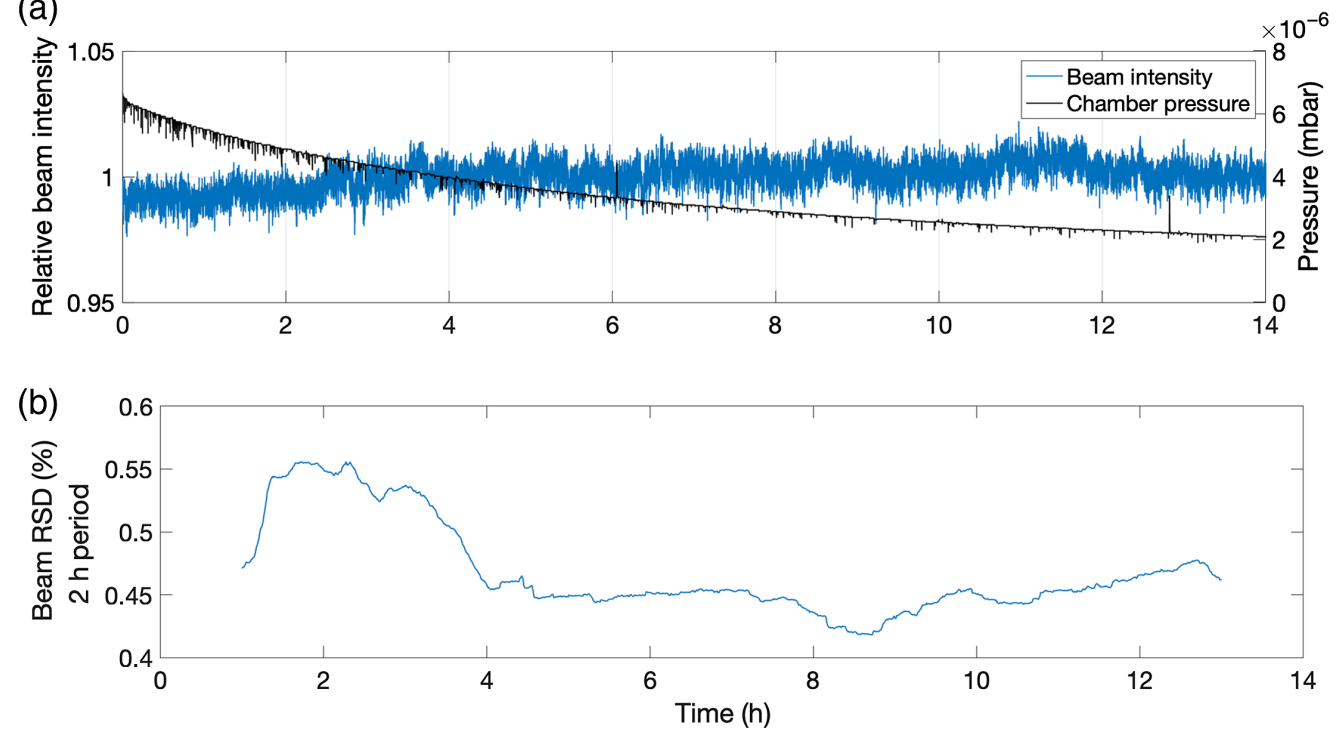

Fig. 6 (a) Evolution of chamber pressure and relative beam intensity measured over a period of $14 \mathrm{~h}$ with a sampling frequency of $1 \mathrm{~s}$. (b) RSD of recorded beam intensity over any $2 \mathrm{~h}$ period within total measurement duration.

that the monochromator is optimized for Bragg reflection of $\mathrm{Al} K_{\alpha}$ at $6.54 \mathrm{deg}$ and assuming the experimentally obtained $\mathrm{Al} K_{\alpha}$ line width $\Delta E \approx 0.85 \mathrm{eV} .{ }^{19}$

$$
\begin{gathered}
m \lambda=2 d \sin \theta \Rightarrow m \frac{d \lambda}{d \theta}=2 d \cos \theta, \\
\Rightarrow d \theta \frac{\lambda}{d \lambda}=\tan \theta_{B, K_{\alpha}} \Leftrightarrow \Delta \theta=\frac{\Delta \lambda}{\lambda} \tan \theta_{B, K_{\alpha}}, \\
\theta_{B, K_{\alpha}}=6.5^{\circ} \Rightarrow \Delta \theta \approx 13.6^{\prime \prime} .
\end{gathered}
$$

The Darwin width (FWHM) at $1.487 \mathrm{keV}$ of the monochromator is $\zeta_{D} \approx 8.1^{\prime}$. The angular acceptance at which a beam of this energy is reflected is thus the convolution $\Delta \theta_{\text {monochromator }}=\sqrt{\left(\Delta \theta_{B, K_{\alpha}}\right)^{2}+\left(\zeta_{D}\right)^{2}} \approx 8.1^{\prime}$. With a horizontal beam divergence of $0.72^{\prime}$ determined by the KB mirrors, this monochromator does not alter the angular resolution of the system. If the position sensitivity capability of the detector is used for example scatter measurements, an angular resolution of $0.74^{\prime}$ is obtained by convoluting the beam divergence with the angular size of the pixels $(26 \mu \mathrm{m})$ as seen from the sample ( $505 \mathrm{~mm}$ away). Slit 3 may be positioned 0 to $220 \mathrm{~mm}$ in front of the sample stage, meaning any opening wider than $0.2 \mathrm{~mm}$ will not further reduce horizontal divergence.

Figure 7 shows a measurement of the scattering from the reflectometer components (collimating mirrors, slits, monochromator, etc.) themselves. The measurement has a FWHM of $0.14 \mathrm{deg}$ but more importantly it shows that instrument scatter is $\mathcal{O}\left(10^{-4}\right)$ or less, meaning the scatter instrument function is negligible during measurements as it folds into the specular reflectivity measurements in the second order. At rocking curve measurements, the specular part of the scattering from the instrument components will be reflected outside the detector when $\omega \gtrsim \pm 0.5 \mathrm{deg}$ from the specular direction. If sample scatter measurements were instead carried out at fixed incidence by scanning the detector angle, a deconvolution between instrument scatter and measured scatter would have to be performed to obtain the scattering from the sample.

The KB mirrors are manufactured to produce a beam of $0.51 \mathrm{~mm}$ width with better collimation than can be obtained by the slits so pre-sample slits are only used to cut away any scattering from reflectometer components and down-shaping the beam if needed. Closely scanning the direct beam with a detector slit width of $0.1 \mathrm{~mm}$, the monochromatized beam is found to have 


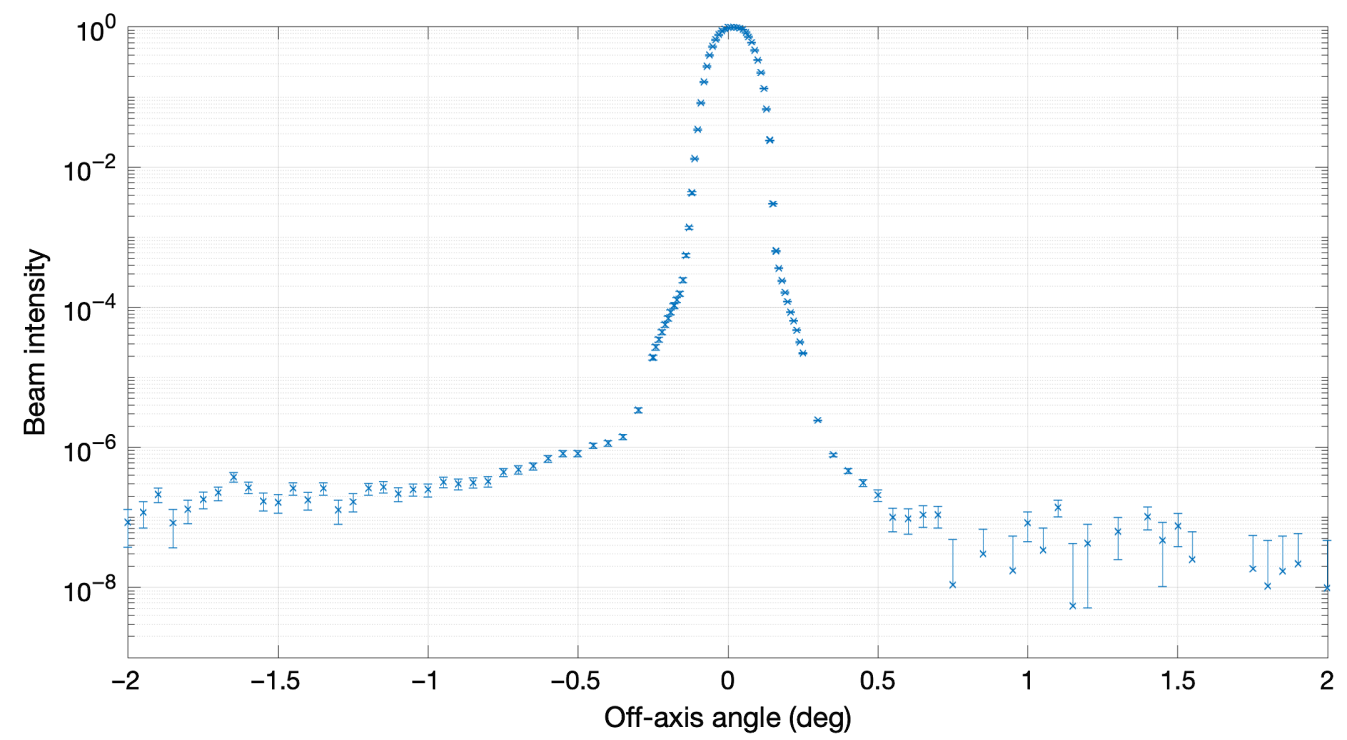

Fig. 7 Measurement of the instrumental scatter function using a pre-sample slit width of $0.7 \mathrm{~mm}$ to ensure encompassing the whole beam (collimated to $0.51 \mathrm{~mm}$ ).

FWHM $=0.115 \mathrm{deg} \Leftrightarrow 1.014 \mathrm{~mm}$ measured at $2 \theta$ in the detector. This corresponds to the beam having a divergence of $0.746^{\prime}$, in perfect agreement with the analytical calculations.

\subsection{Detector and Dynamic Range}

LEXR is equipped with a position sensitive CCD detector that is used for alignment of reflectometer components and samples, as well as for data acquisition. Its specifications are listed in Table 2.

The noise level of the detector is highly dependent on temperature. The detector is actively cooled by a Peltier element. At high temperatures, the total noise is dominated by dark current and is thus proportional to exposure time. When cooled, the readout noise is dominant, effectively making the total noise almost independent of exposure time. Figure 8 shows measurements of the noise as function of temperature and exposure time at different readout

Table 2 Specifications of greateyes GE-VAC 1024256 BI DD detector. ${ }^{20}$ Although the detector has a larger image area, the detector slit limits the throughput to a $7 \times 7 \mathrm{~mm}^{2}$ area.

\begin{tabular}{lc}
\hline \hline Detector specification & Value \\
\hline Pixel format & $1024 \times 256$ \\
Image area & $26.6 \mathrm{~mm} \times 6.7 \mathrm{~mm}$ \\
Pixel size & $26 \mu \mathrm{m} \times 26 \mu \mathrm{m}$ \\
Energy range & 10 to $11 \mathrm{keV}$ \\
Typ. read noise at $500 \mathrm{kHz}$ & $9.7 \mathrm{e}^{-}$ \\
Dark current at $-80^{\circ} \mathrm{C}$ & $0.08 \mathrm{e}^{-} / \mathrm{pixel} / \mathrm{s}$ \\
Quantum efficiency at $1.487 \mathrm{keV}$ & 0.87 \\
Photoelectrons/photon at $1.487 \mathrm{keV}$ & 400 \\
Quantum efficiency at $8.048 \mathrm{keV}$ & 0.45 \\
Photoelectrons/photon at $8.048 \mathrm{keV}$ & 2000 \\
\hline \hline
\end{tabular}



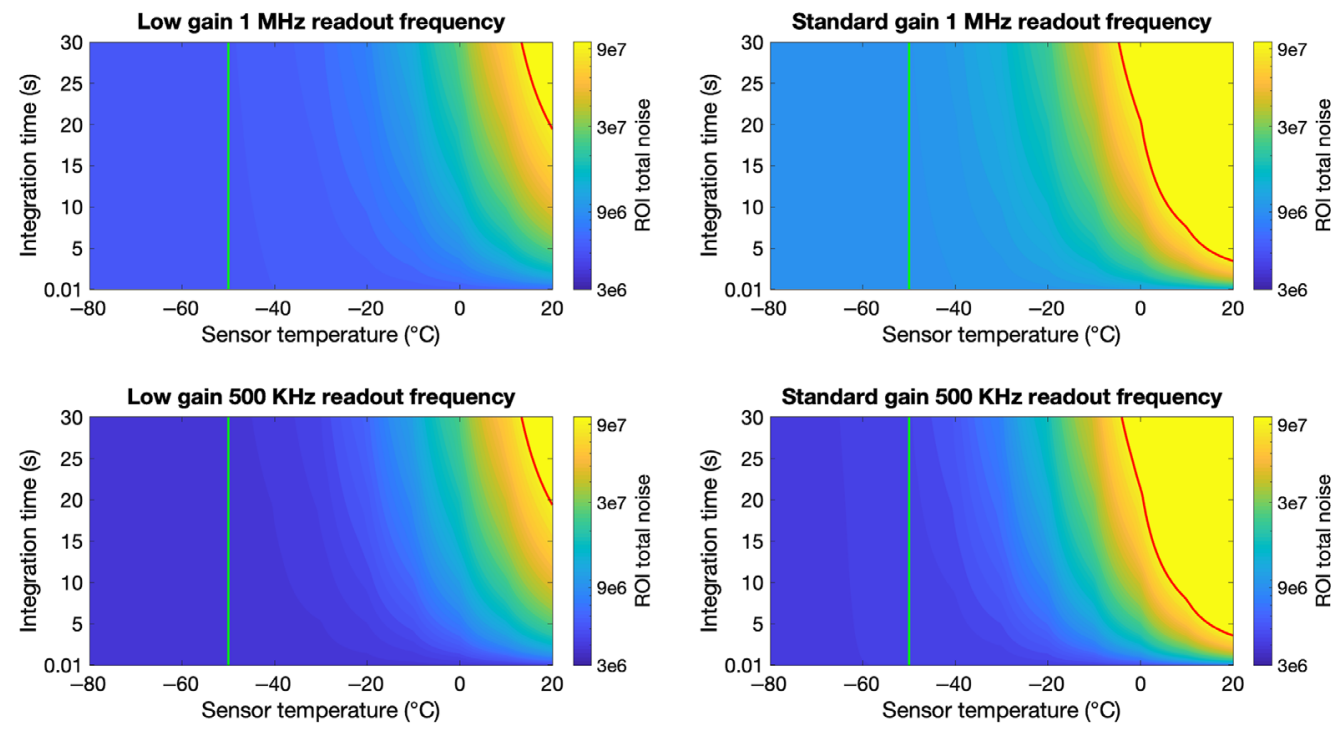

Fig. 8 Total integrated noise in a suitable ROI $(80 \times 110$ pixels), each plot shows a natural cubic spline interpolation from $11 \times 12$ data points. The red line marks the point of detector saturation. The green line indicates the sensor temperature of $-50^{\circ} \mathrm{C}$ used for standard measurements.

frequencies and gain settings. From these measurements, it is decided to use a standard temperature setting of $-50^{\circ} \mathrm{C}$ as a trade-off between a noise level close to independence of exposure time, whereas at the same time minimizing strain on the Peltier element. The noise obtained in a region of interest (ROI) is independent of any light source on neighboring pixels in the detector. In addition to the options shown, a readout frequency setting of $3 \mathrm{MHz}$ is used only for alignment purposes.

The flux of $\mathrm{K}_{\alpha}$ photons from the $\mathrm{Al}$ source is from simulations expected to be $8 \times 10^{7}$ photons/s after the KB mirrors. For typical measurements, the beam is downshaped from $0.51 \times 2.5 \mathrm{~mm}^{2}$ to $0.5 \times 2 \mathrm{~mm}^{2}$, although this has small impact on the flux as it merely cuts the tails off the beam profile in either direction. With the aforementioned reflectance of the monochromator and $78 \%$ combined throughput of the attenuation filters at $1.487 \mathrm{keV}$, the expected number of photons impinging on the detector in the free beam (without mirror samples in place) is thus around $2.8 \times 10^{7}$ photons/s.

For the ROI of Fig. 8, 8800 pixels are read out so an integration time of, e.g., $3 \mathrm{~s}$ will result in a total noise of $\sim 8.7 \times 10^{4} \mathrm{e}^{-}$. Each absorbed 1.487-keV photon generates about 400 photoelectrons and the detector quantum efficiency is $87 \%$ at this energy so a flux of $2.8 \times 10^{7}$ photons $/ \mathrm{s}$ yields a $\mathrm{S} / \mathrm{N} \approx 3.4 \times 10^{5}$. This ratio scales almost linearly with exposure time as the dark noise is a subordinate noise contributor. During specular measurements exposure times of up to $30 \mathrm{~s}$ at each data point are used yielding a dynamic range of more than six orders of magnitude. Exposure times of $300 \mathrm{~s}$ and minimization of the ROI allows eight orders of magnitude to be reached as demonstrated on the non-specular scatter measurement of an Ir coated NuSTARtype $^{21}$ mirror in Fig. 9.

\section{System Utilization and Characteristics}

\subsection{Measurement Uncertainty and Repeatability}

The processing of LEXR data includes evaluation of the statistical uncertainty of each data point and appropriate error propagation through background subtraction and data normalization. Systematic uncertainties and measurement errors are, however, difficult to quantify as they are highly dependent on beamline operator precision in sample mounting, alignment, and general operation of the reflectometer. As part of a repeatability study, a sample has been measured 10 times successively and the data is overplotted in Fig. 10. 


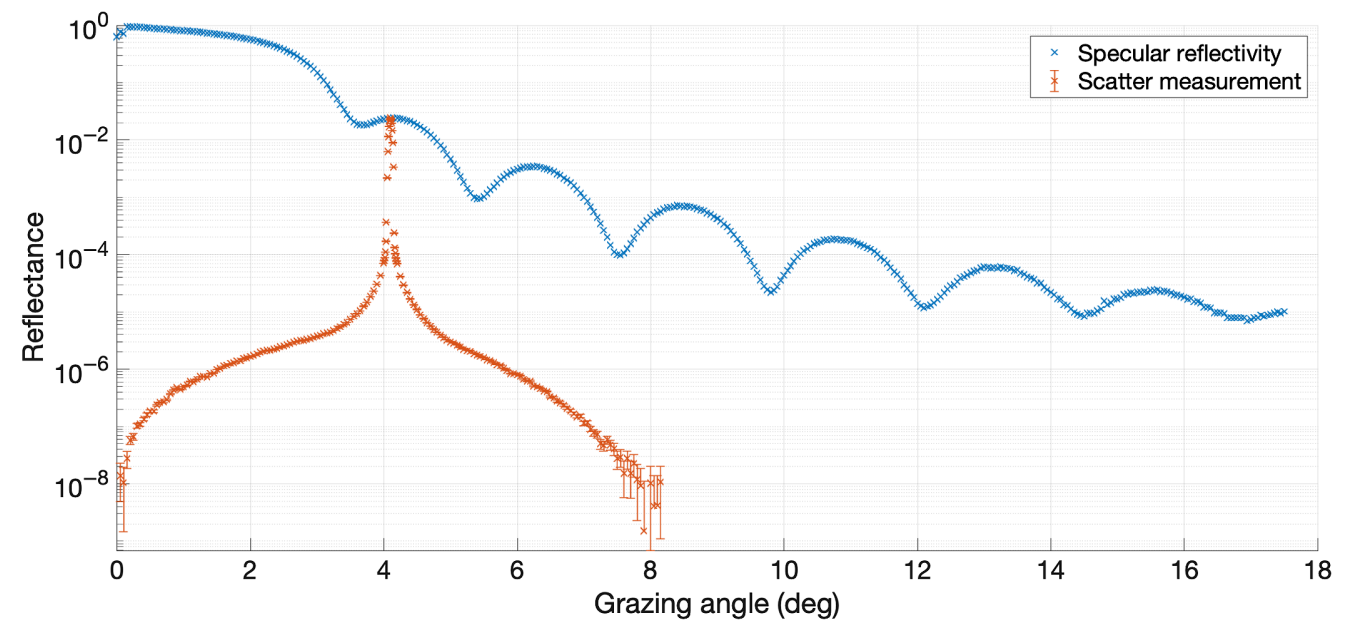

Fig. 9 Specular and non-specular reflectivity measurements of a NuSTAR-type mirror substrate coated with $10 \mathrm{~nm}$ Ir.
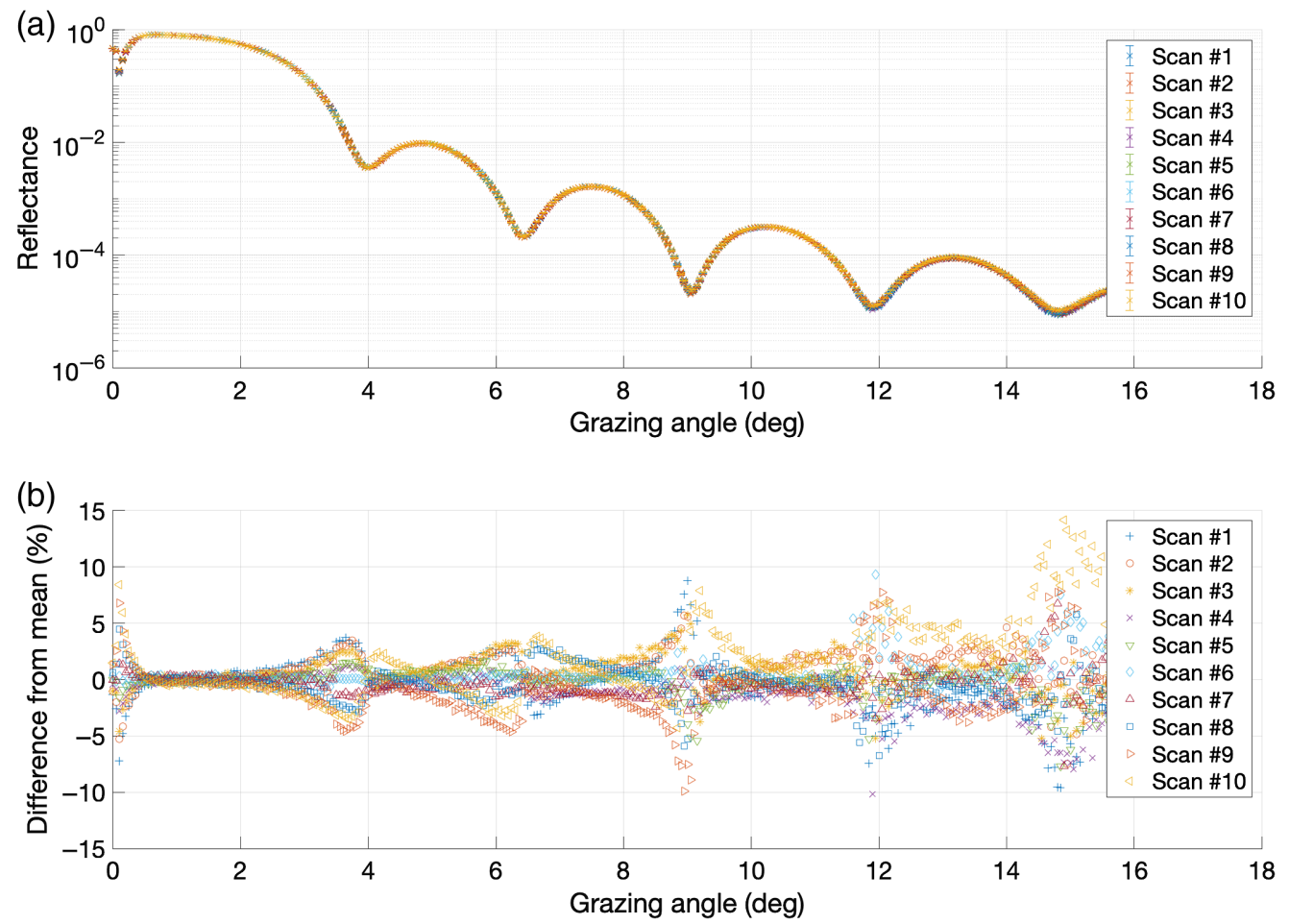

Fig. 10 (a) 10 repeated $\theta-2 \theta$ measurements of the same Ir single layer sample. (b) Variation in percent from mean value of reflectance at each incidence angle. The data is in statistical agreement but error bars have been omitted here for clarity.

The spread of measured reflectance curves follows a pattern that is inversely correlated with the oscillations of the Kiessig fringes, indicating that one contributor to the variation in measured reflectance is statistical or signal dependent rather than inconsistency in alignment or motor control. Furthermore, the spread in the difference from the mean is centered around 0 , meaning that no single measurement deviates substantially from the other nine. There is an increase in variation at the bottom of Kiessig fringes as the signal/noise decreases and, indeed, the largest discrepancies are found at higher grazing angles when the level of noise dominates the measured data. The sawtooth pattern is, however, a clear indication that the main contributor to the 

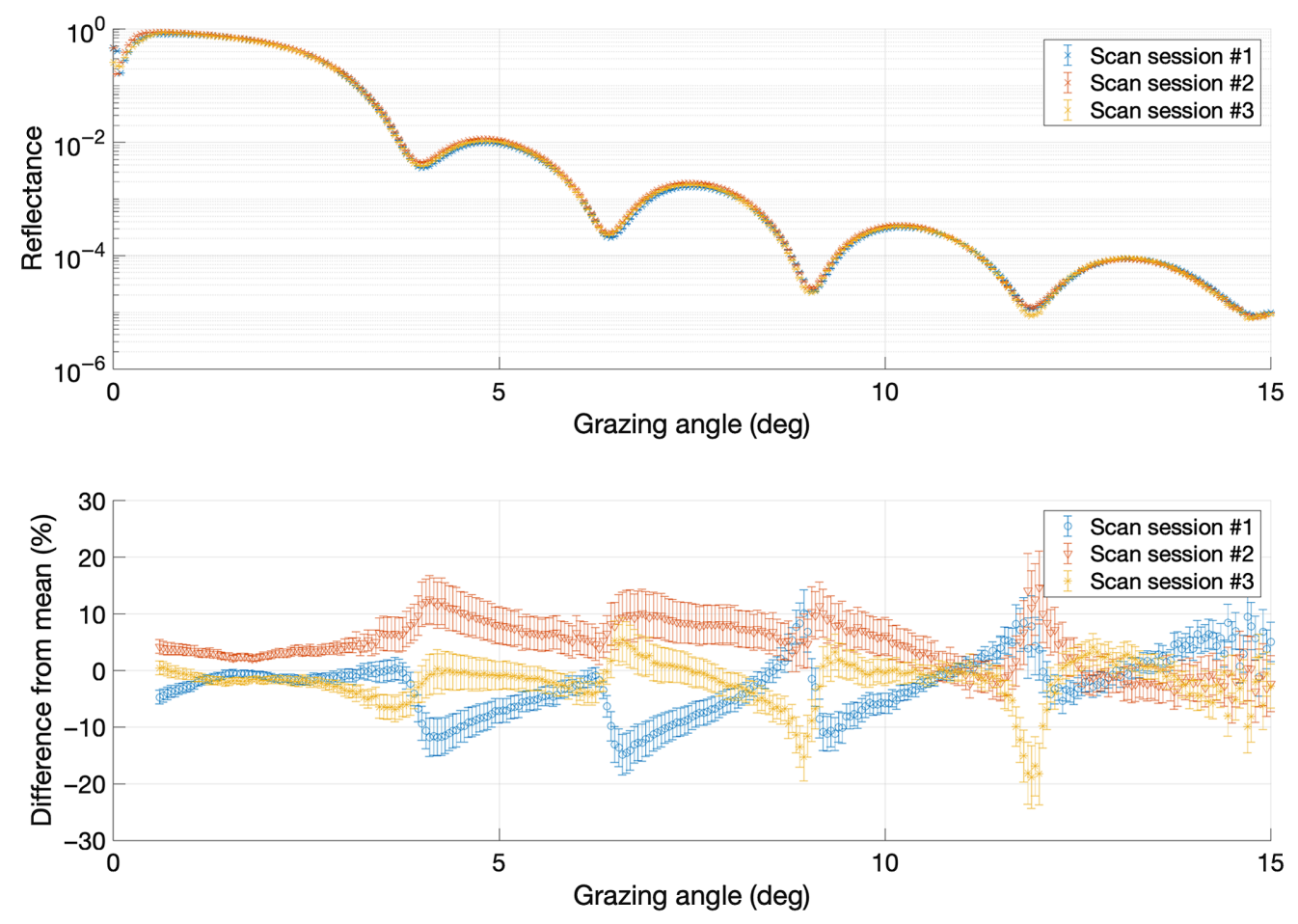

Fig. 11 Measurements of the same sample in three different sessions, meaning that the vacuum chamber was vented, the sample remounted in a different position on the sample holder, realigned and remeasured. The difference from the mean below the angle at which the full beam footprint is on the sample has been omitted.

difference from the mean is a shift in angle due to motor backlash rather than a signal strength variation.

Figure 11 shows repeated measurements of the same sample where the vacuum chamber was vented between each measurement, the sample remounted in a different position on the sample holder, realigned in the new position, and measured with different source and detector settings. The repeated measurements again show very similar reflectivity curves, albeit with a larger variation than for measurements performed repeatedly in the same session. It is clear from the difference from the mean of the three measurements that varying the measurement settings result in different levels of uncertainty. Evident from the region of total external reflection, there are systematic uncertainties of $\lesssim 3 \%$ that are unaccounted for and the slope and sawtooth pattern of the difference curves again show that the main contributor to the difference is the non-identical alignment. The much larger difference between the measurements compared to those shown in Fig. 10 is thus due to systematic errors in mounting and alignment precision whereas the previous repeated measurements only had a small systematic shift due to backlash, which is an order of magnitude smaller. The best-fit parameters obtained to these measurements are compared in Fig. 12. For the ten consecutive measurements, a small drift from one measurement to the next occurs while remounting and realigning a sample exemplifies the effect of sample misalignment on the systematic uncertainty of the system and the best-fit parameters to the acquired data.

While the thicknesses of the Ir coating and hydrocarbon overlayer are almost identical in all fits, the roughness parameters vary slightly but largely remain within $\pm 3 \%$. Some systematic error can be seen from the fitted density of the hydrocarbon overlayer. The best fits show a trend of slightly increasing density for each measurement in the same session. This is due to the high sensitivity of this parameter to the critical angle so a slight shift in motor positions due to e.g. backlash when resetting for the next measurement will shift the alignment angle of the sample in the same direction by a small amount. Interpolation from the data shows a maximum difference on angular alignment of $13.3^{\prime}$ with a mean difference between each repeated measurement of $4.3^{\prime \prime}$. The two measurements that were carried out after remounting and manually realigning the 


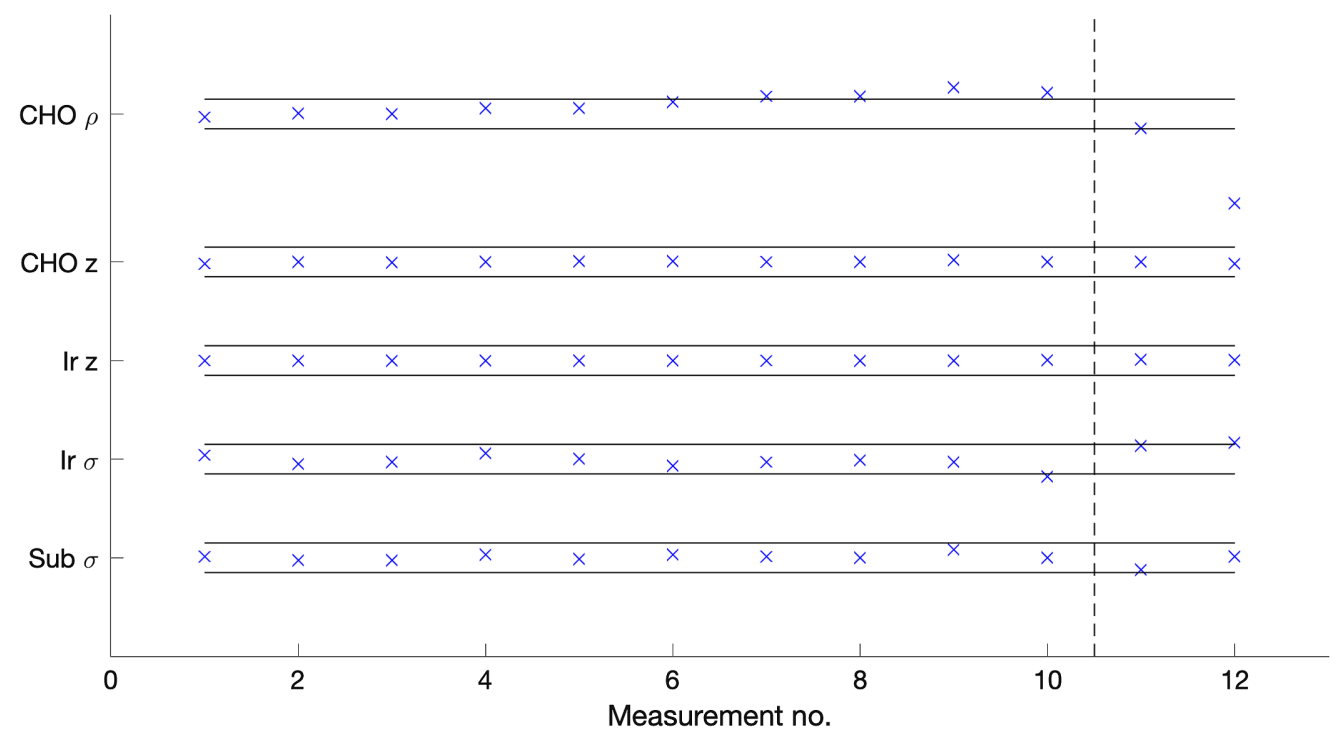

Fig. 12 Comparison of best-fit parameters from the repeated measurements of the same sample. Measurements 1 to 10 were made in succession. Measurements 11 and 12 were each made after remounting and realigning the sample. All points were normalized to the mean obtained value. The black lines indicate $\pm 3 \%$ of the mean fit parameter. $\mathrm{CHO}$ indicates hydrocarbons present on the surface. This could be any $\mathrm{C}-\mathrm{H}-\mathrm{O}$ compound or other contaminants but as this energy is not near any absorption edges, the chemical composition folds into the density parameter.

sample have nearly the same fitted parameters except for the hydrocarbon density, which is off by $5 \%$ and $20 \%$, corresponding to roughly 0.1 and $0.3 \mathrm{~g} / \mathrm{cm}^{3}$, respectively. This highlights how sensitive fitting parameters, such as density that relies heavily on the critical angle, are to sample alignment. Other parameters such as coating thickness and roughness have absolute variation in fit parameters of $<0.1 \AA$, effectively making these small differences in reflectivity curves negligible. The systematic uncertainty originating from alignment variation cannot be quantified as the alignment accuracy obtainable is both sample and user dependent.

\subsection{Small-Beam Intensity Variation}

The beam cross section is rather non-uniform with the largest pixel-to-pixel variations being around 30\% near the sides (see Fig. 13) and 10\% to 15\% near the center of the beam.

This is irrelevant for measurements where a large portion of the beam is used and the full beam footprint is on the sample. For pencil-beam measurements, however, the slits are reduced so as to only choose a small subsection of the beam cross section and variation in the integrated flux therefore induces large uncertainty in the measured data. Figure 14 shows the direct beam intensity as function of time for different slit sizes.

Variations of the same magnitude are observed for exposures with open slits but where subsections of pixels are chosen in post-processing to match the size of the slits used to obtain Fig. 14. The cause of the variation is thus either constant minor flux variations across the beam cross section, or vibrations induced on the reflectometer from e.g. the turbo pump causing shifts in the flux distribution map. In any case this effect is averaged out so beams larger than $0.2 \mathrm{~mm}^{2}$ have integrated flux variation $<1 \%$.

\subsection{Sample Modeling and Bandwidth Limit on Roughness}

A more natural domain to work in when considering geometric effects is the reciprocal (Fourier) space, in which realspace sample features give rise to a wavelength-independent intensity distribution. For a beam with wavevector $\vec{k}$ reflecting elastically off a sample with angle $\theta$, it can be seen (Fig. 15) that the momentum transfer vector is $q_{\|}=4 \pi \sin (\theta) / \lambda$. For single layers of 

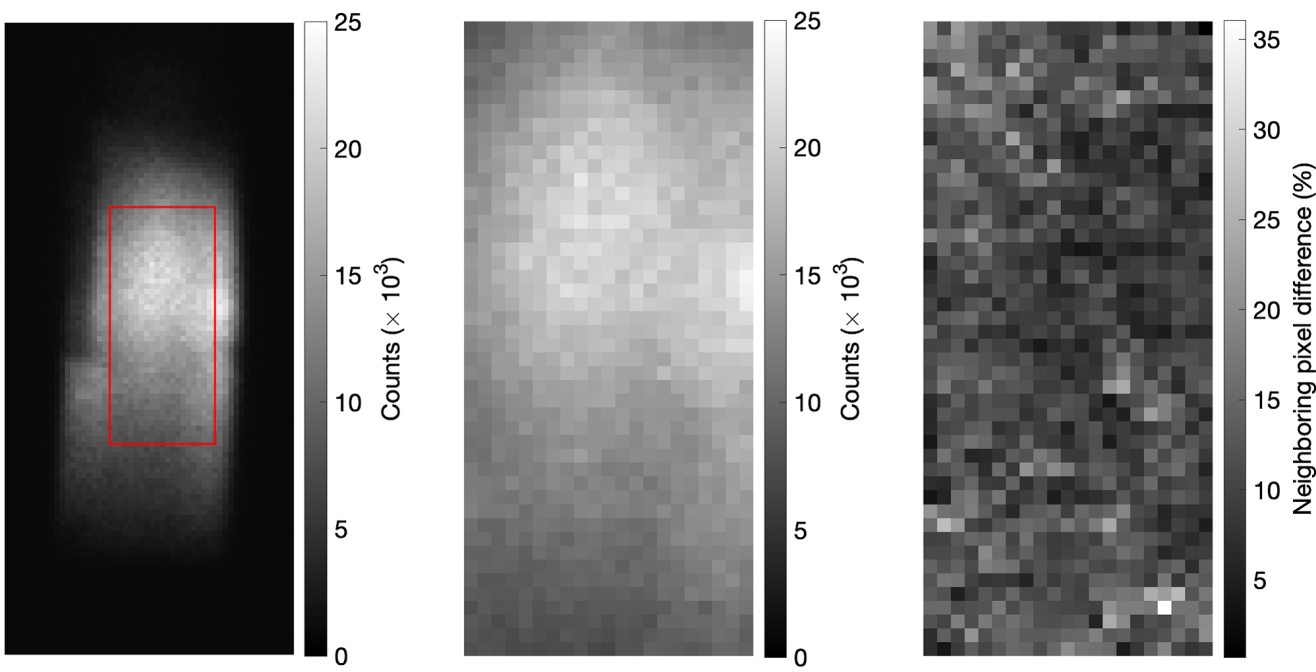

Fig. 13 Left: Full beam as seen from the detector. Mid: Subsection of beam, corresponding to red box on left image, ensuring the beam edge is excluded. Right: Largest signal strength difference to any of the eight neighboring pixels within the beam subsection.

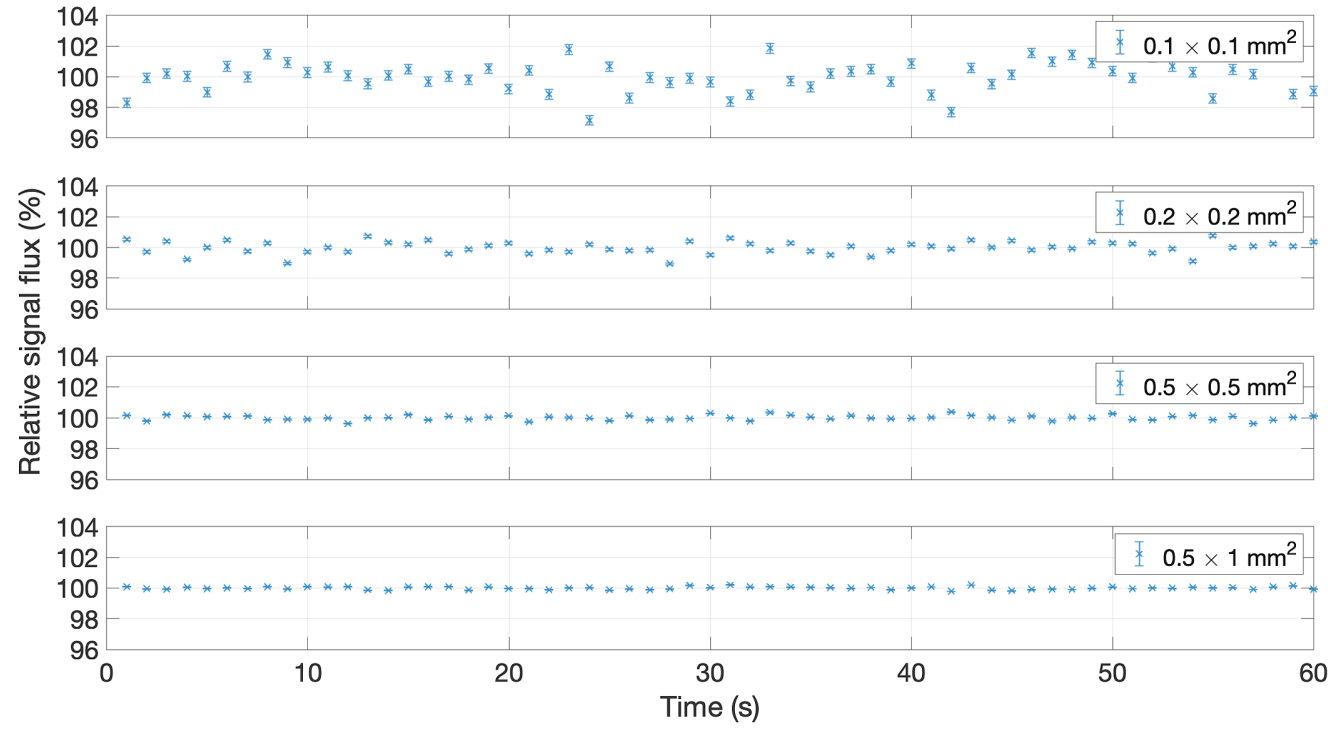

Fig. 14 Variation of beam intensity relative to the mean over $60 \mathrm{~s}$ with a $1 \mathrm{~s}$ sampling frequency for different slit sizes.

low-density materials or uncoated substrates, the limited dynamic range of our $8.048 \mathrm{keV}$ system means that LEXR can probe a larger part of reciprocal space.

\subsection{Reciprocal Space Limitation on Determination of Low-Density Overlayers}

Simulations show (Fig. 16) that a smooth hydrocarbon overlayer on $10 \mathrm{~nm}$ Ir will give rise to interference structures in the region $q_{\|} \sim 8-10 \mathrm{~nm}^{-1}, 7-8$ orders of magnitude down, corresponding to $5.6 \mathrm{deg}$ to $7 \mathrm{deg}$ at $8.048 \mathrm{keV}$ or $32 \mathrm{deg}$ to $42 \mathrm{deg}$ at $1.487 \mathrm{keV}$. This is beyond the mechanical range of LEXR and beyond the dynamic range of our available $8.048-\mathrm{keV}$ reflectometer. This effect should, however, be obtainable from synchrotron measurements.

The dynamic range of the $8.048-\mathrm{keV}$ system at DTU Space is $5 \times 10^{-6}$ and the vacuum chamber geometry of LEXR effectively limits its reciprocal spatial range to $4.5 \mathrm{~nm}^{-1}$. Other 

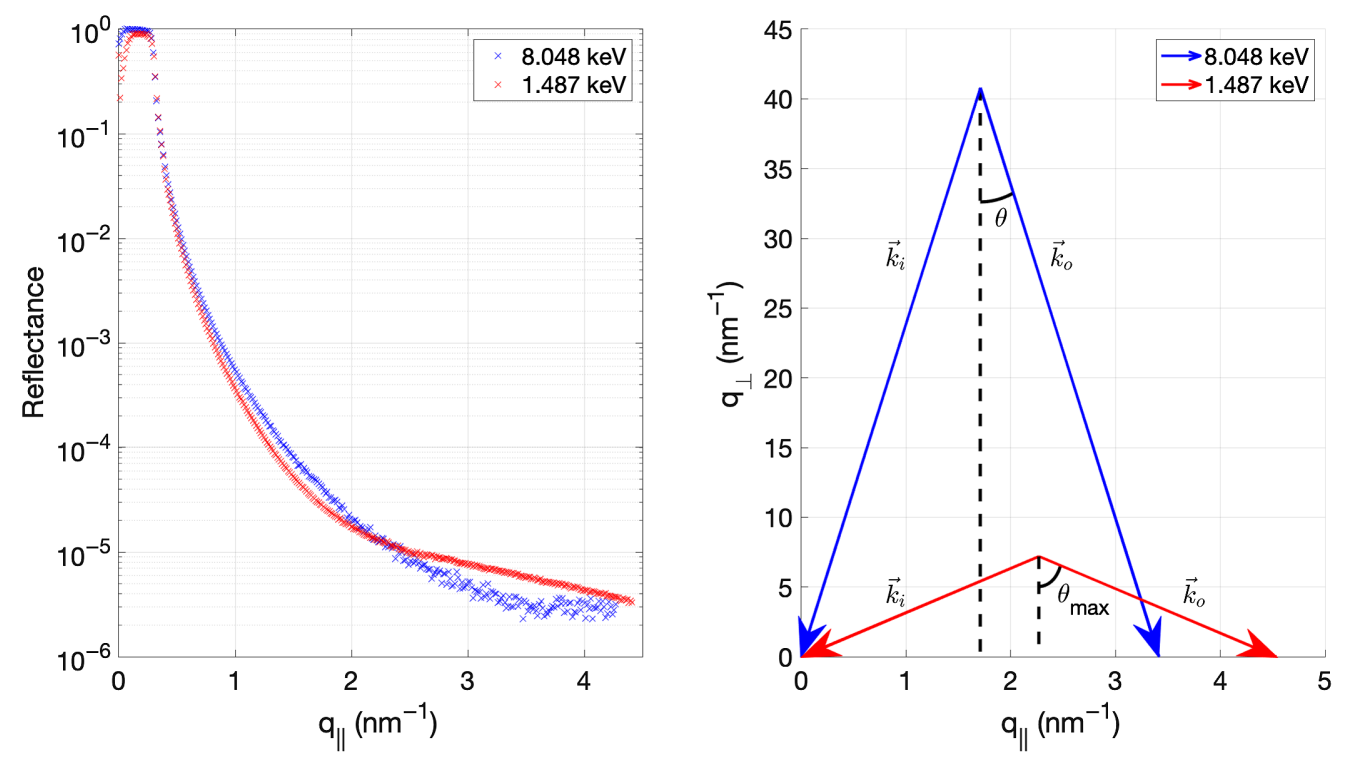

Fig. 15 Left: Reflectivity measurements of uncoated substrates plotted in q-space. Right: Illustration of momentum transfer vectors for the two measurements. The angles are exaggerated due to the different axis limits. The $8.048 \mathrm{keV}$ measurement is limited to $q_{\|} \approx 3.4 \mathrm{~nm}^{-1} \Leftrightarrow 2.4 \mathrm{deg}$ by the dynamic range, whereas the $1.487 \mathrm{keV}$ measurement is limited by chamber geometry to $\theta_{\max }=17.5 \mathrm{deg} \Leftrightarrow 4.53 \mathrm{~nm}^{-1}$.

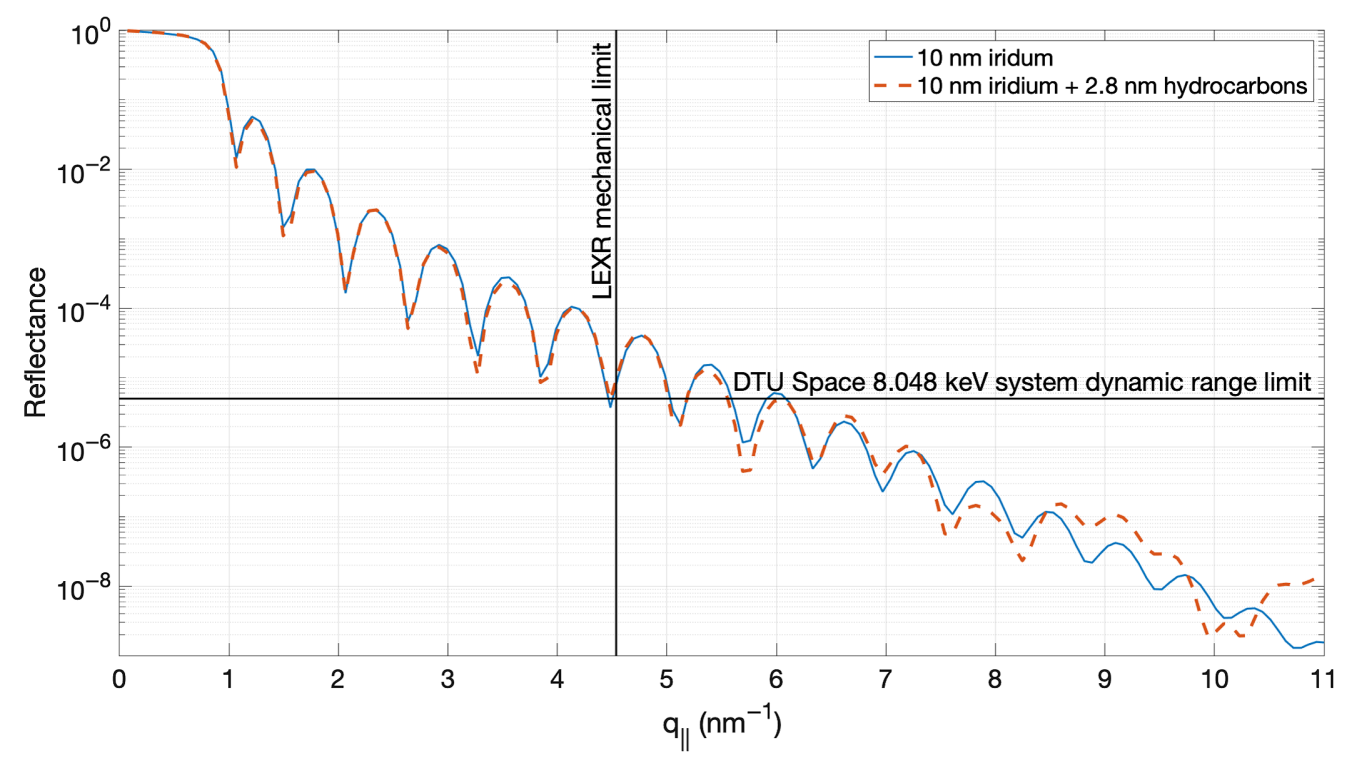

Fig. 16 Simulated effect on reflectance of a smooth hydrocarbon overlayer on $10 \mathrm{~nm}$ Ir plotted in reciprocal space. It is worth noting from the limits of the two reflectometers that the interference region 8 to $10 \mathrm{~nm}^{-1}$ cannot be reached by either system.

than this purely geometric consideration, low-density overcoats such as naturally occurring hydrocarbons do, however, also alter the shallow-angle reflectance, an effect not present at higher energies and one that is being utilized by purposeful deposition of low-Z materials to increase reflectance. ${ }^{22,23}$ This effect is the reason the curves in Fig. 15 (left) do not overlap entirely and the consequence is that hydrocarbons can be modeled and must be accounted for in LEXR measurements as shown in Fig. 17. 

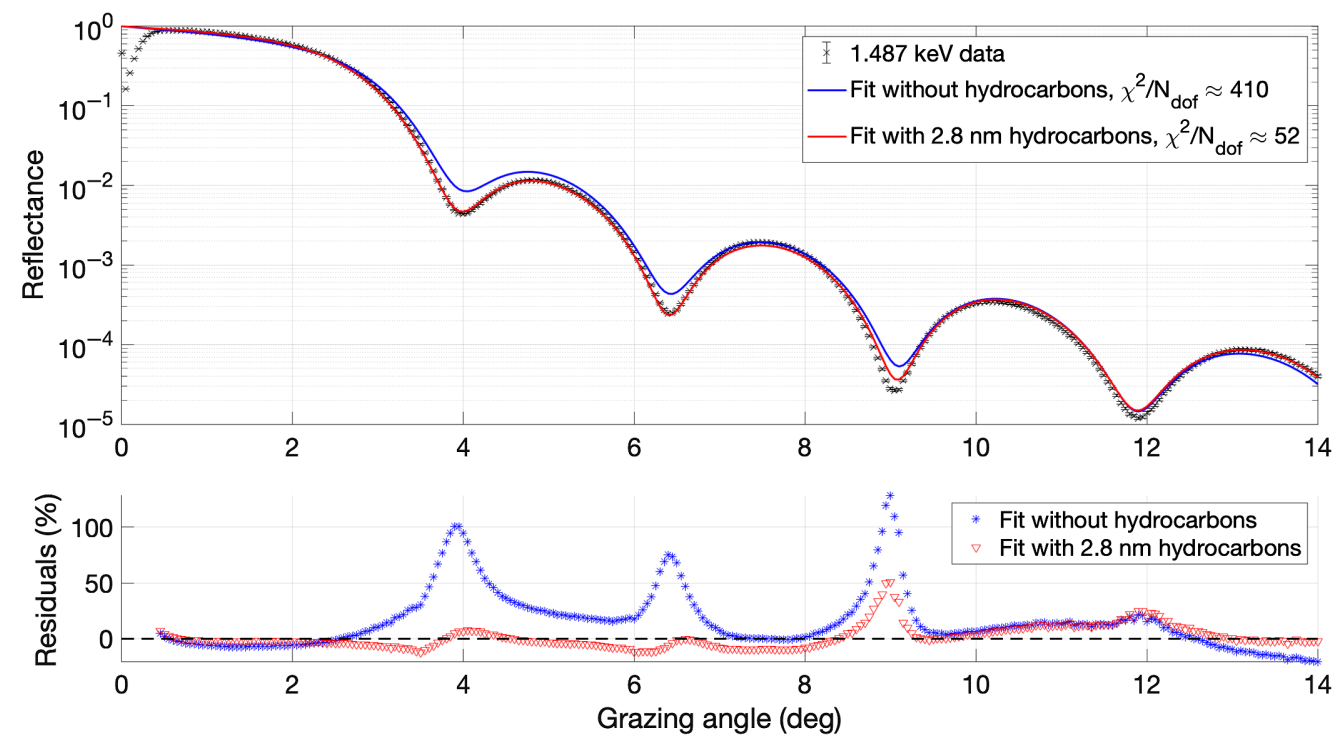

Fig. 17 Comparison of fits to a single layer Ir coated sample using models with and without hydrocarbon overlayers.

Due to the measurement errors only accounting for statistical and not systematic uncertainties, the errors are underestimated so even for the better fit, the reduced $\chi^{2}$ is rather large. The method can nevertheless be used to compare several models and determine which one is better for the sample in question. Adding an uncertainty of around 3\% of the signal as discussed in relation to Fig. 11, the reduced $\chi^{2}$ becomes 6 .

\subsection{Bandwidth Limit on Roughness Determination}

Typically, measurements are performed with an ROI spanning 80 pixels in width, which, with a pixel pitch of $26 \mu \mathrm{m}$, corresponds to around $2 \mathrm{~mm}$. With a sample-to-detector distance of $505 \mathrm{~mm}$, the maximal scatter acceptance angle is around $\Delta \theta_{s} \approx 7$ arcmin. From the more detailed scatter geometry illustrated in Fig. 18, it can be seen that, generally, the width of the scatter distribution follows

$$
\Delta \theta \approx \frac{2 \lambda}{\xi \sin \left(\theta_{i}\right)}
$$

where $\xi$ denotes the lateral correlation length of the sample roughness. As the incidence angle $\theta_{i}$ decreases, the scatter angle eventually becomes parallel with the mirror surface, after which the width of the distribution becomes independent of incidence angle $\Delta \theta \approx \sqrt{2 \lambda / \xi}$.

For LEXR with $\lambda \approx 8.34 \AA, \Delta \theta_{s} \approx 7 \operatorname{arcmin}$, and $\theta_{i}=5 \mathrm{deg}$ we have $\xi \approx \lambda /\left(\Delta \theta_{s} \theta_{i}\right) \approx$ $4.7 \mu \mathrm{m}$ meaning that fitting to the specular measurement around 5 degrees will give information about the roughness with $\xi<4.7 \mu \mathrm{m}$ and, more generally, information can only be extracted about features with correlation lengths no larger than a few tenths of microns, as the scatter angles are otherwise sufficiently small to fall within the measured specular beam. Alternatively, the detector acceptance angle can be varied during the scan such that the probed correlation lengths remain constant. Investigating, for example, the roughness region with $2800 \mathrm{~nm} \geq$ $\xi \geq 800 \mathrm{~nm}$, one could compare reflectivity measurements where the detector opening varies from fully open down to 0.8 and $2.8 \mathrm{~mm}$ at $17.5 \mathrm{deg}$, respectively. The detector slit is placed $410 \mathrm{~mm}$ from the sample and its maximal width of 7 -mm limits the accepted total scatter width to $\Delta \theta_{\max } \approx 58.7$ arcmin, which in turn limits the correlation length to $\xi \sin \left(\theta_{i}\right) \gtrsim 97.6 \mathrm{~nm}$.

Carrying out such a measurement on a sample with a $10-\mathrm{nm}$ Ir coating on a NuSTAR-type glass substrate that also includes a "standard scan" of constant $2 \mathrm{~mm}$ acceptance width on the detector, we find that the measured reflectance falls slightly below the others on the standard 


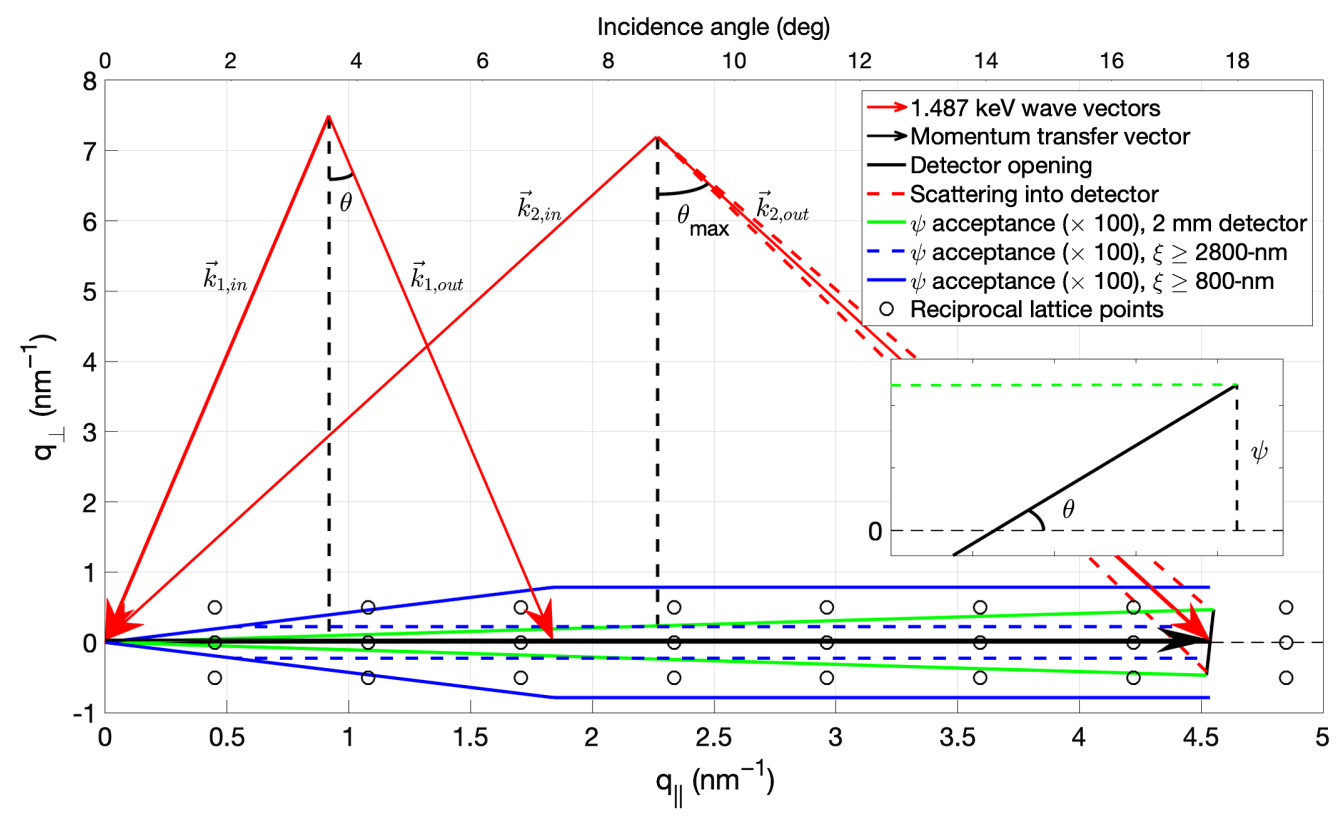

Fig. 18 Illustration of the LEXR scattering geometry in reciprocal space. $\vec{k}_{\text {in }}$ and $\vec{k}_{\text {out }}$ are the incoming and outgoing wave vectors. $\theta$ is the beam incidence angle on the sample and $\psi=2 \pi / \xi$ is the reciprocal transverse characteristic length. The $\psi$ acceptance region is indicated for a detector of fixed width throughout the measurement as well as where the detector acceptance width is varied to probe a constant range of correlation lengths. The angles are exaggerated due to the different axis limits. The spacing of reciprocal lattice points along $q_{\|}$are those of a 10-nm coating, their spacing in $q_{\text {perp }}$ is arbitrary. The insert shows the geometry of the transverse probing length at any point during the measurement.

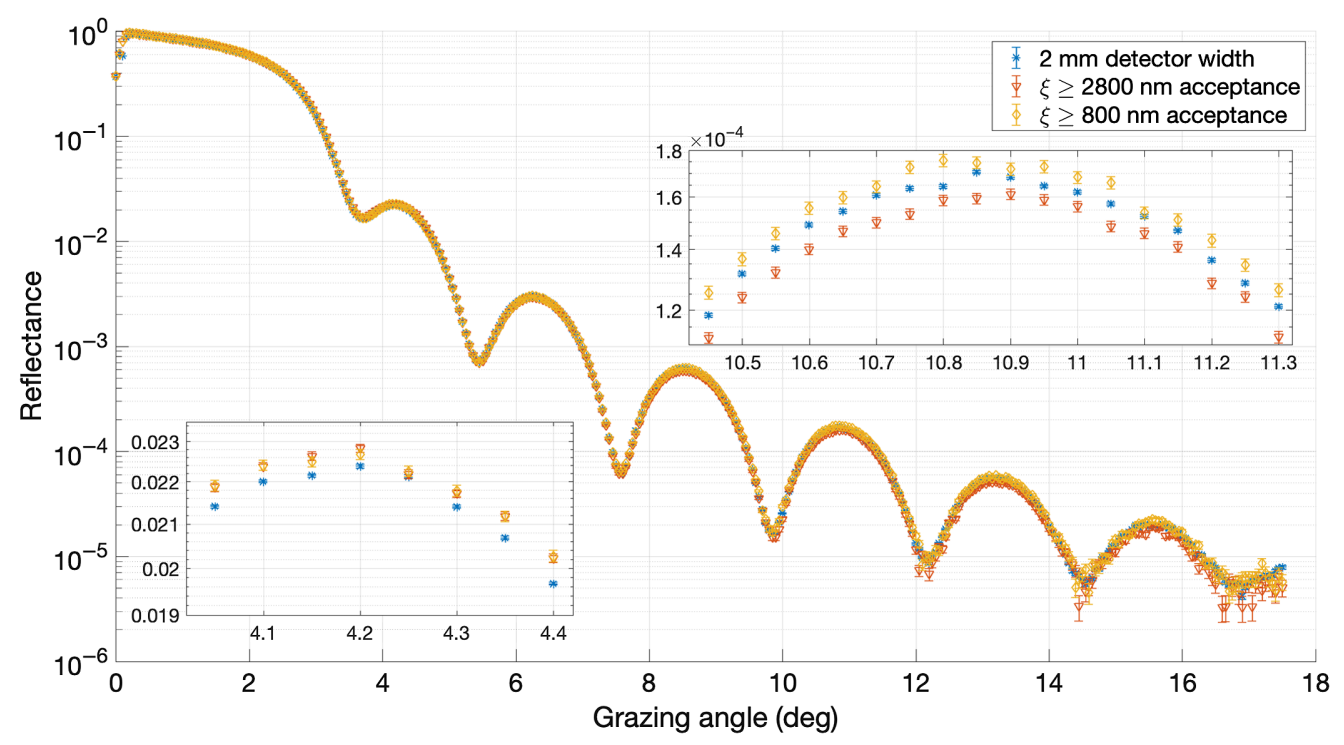

Fig. 19 Repeated measurements of a NuSTAR-type glass substrate coated with 10-nm Ir with different acceptance widths at the detector. Inserts show close-ups of two parts of the scan.

scan up to around $5 \mathrm{deg}$ (shown in Fig. 19). From around $7 \mathrm{deg}$ to $8 \mathrm{deg}$, the measured intensity on the $2800 \mathrm{~nm}$ scan falls below the other scans, which is also where the two $\psi$ acceptance curves intersect as indicated on Fig. 18. From this point on there is a clear, albeit small $(\sim 8 \%)$, difference such that $\mathrm{R}(\xi \geq 800 \mathrm{~nm})>\mathrm{R}(2 \mathrm{~mm}$ acceptance $)>\mathrm{R}(\xi \geq 2800 \mathrm{~nm})$ until around 


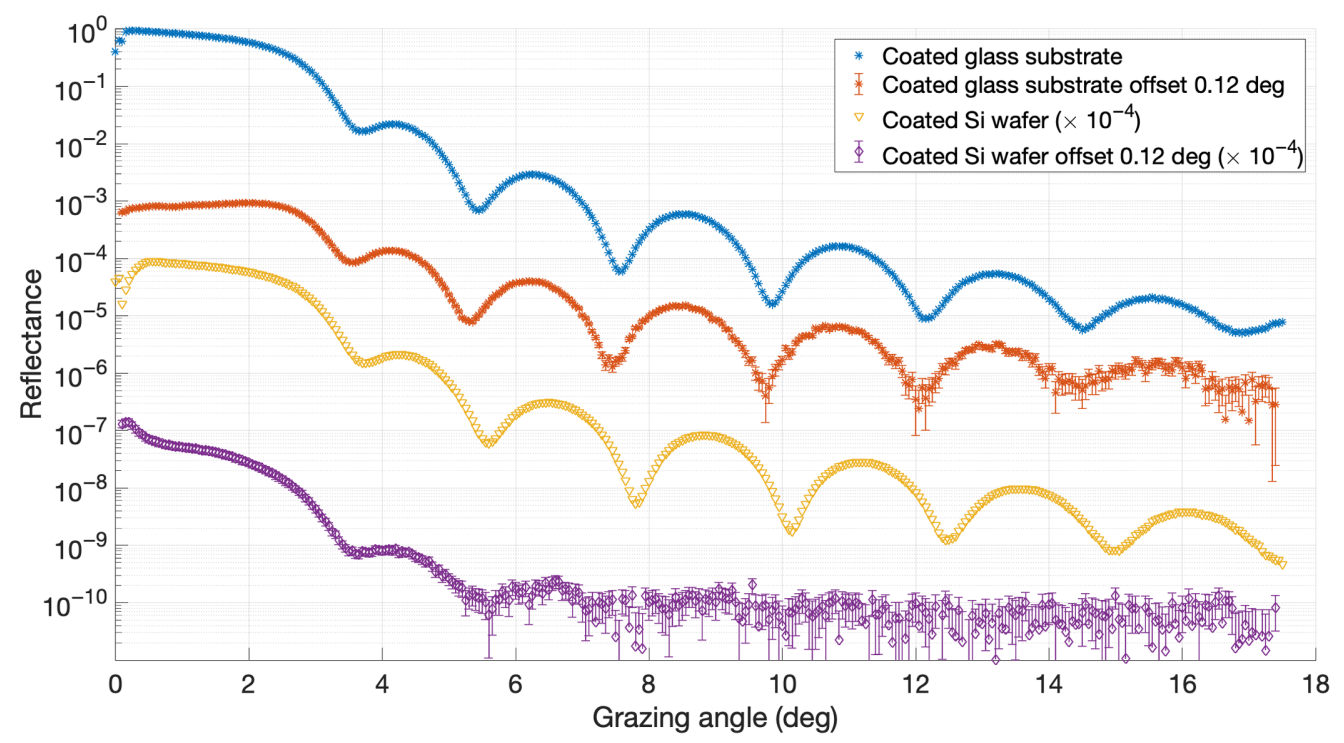

Fig. 20 Specular and offset specular measurements on NuSTAR-type glass substrate and flat Si wafer coated with $\sim 10 \mathrm{~nm} \mathrm{Ir}$.

$\theta_{i} \sim 13.5 \mathrm{deg}$ from which point on $\mathrm{R}(\xi \geq 800 \mathrm{~nm}) \approx \mathrm{R}(2 \mathrm{~mm}$ acceptance $)>\mathrm{R}(\xi \geq 2800 \mathrm{~nm})$, indicating that the sample has at least one roughness correlation length $\xi \approx 1.7 \mu \mathrm{m}$.

Performing the same type of measurements on a flat $\mathrm{Si}$ wafer coated with $10 \mathrm{~nm} \mathrm{Ir}$, the three measurements agree to within the statistical uncertainty of the measurements, indicating an exclusion region $\xi<800 \mathrm{~nm} \vee \xi>2800 \mathrm{~nm}$. Furthermore, the agreement of the reflectivity measurements for different ROIs on the detector verifies the background subtraction method.

A reflectance measurement with the sample and detector moving with the ratio 1:2 but with the sample stage slightly offset from zero gives a measurement along $q_{\|}$but offset in $q_{\perp}$. This probes the scattering component close to the specular direction, which is assumed to be similar to the amount of scattering hidden within the specular beam. In principle, this should be subtracted from the first measurement to remove the component of scattered light hidden within the specular beam but as shown in Fig. 20, this effect is sufficiently small to be negligible in data fitting. The scattering from Kiessig fringes gives rise to streaks out to $2 \pi / \xi$ in $q_{\perp}$ and these are clearly recorded on the measurement of the coated glass substrate where the specular peaks are echoed in the offset-specular measurement. This measurement is offset by about the same angle as the detector acceptance width at high angles and the $\xi \geq 800 \mathrm{~nm}$ scan has a width about twice as large as the standard scan. We see that, indeed, the difference between these two scans on Fig. 19 (see top right insert) is the same order of magnitude as the amount of scattering measured at the offset (orange curve in Fig. 20). The offset specular measurements also agree with the equivalence of the three types of measurements on the coated Si wafer as the offset measurement here indicates only very large-scale roughness correlation on the order of several tens of micrometers.

By inserting the Bragg equation into Eq. (4), one can see that $\Delta \theta \propto d / \xi$ independent of wavelength. Bragg features occur at larger angles for lower energies so a larger $\Delta \theta$ is available before shadowing effects from the sample appear, meaning that for non-specular scatter measurements, low-energy reflectometers can give information about smaller $\xi$.

\subsection{Comparison with BESSY II Synchrotron Beamline}

To compare with $1.487 \mathrm{keV}$ measurements from another beamline, LEXR measurements were carried out on two samples previously measured at the Optics Beamline at BESSY II synchrotron ${ }^{24}$ and reported in Ref. 25. Figure 21 shows the data from BESSY, overplotted with LEXR data, and the best-fit parameters are listed in Table 3.

The measurements, and consequently the fit parameters, are in good agreement. The small deviation between LEXR and BESSY data on cs00002 in the region 4 deg to 6 deg is expressed 

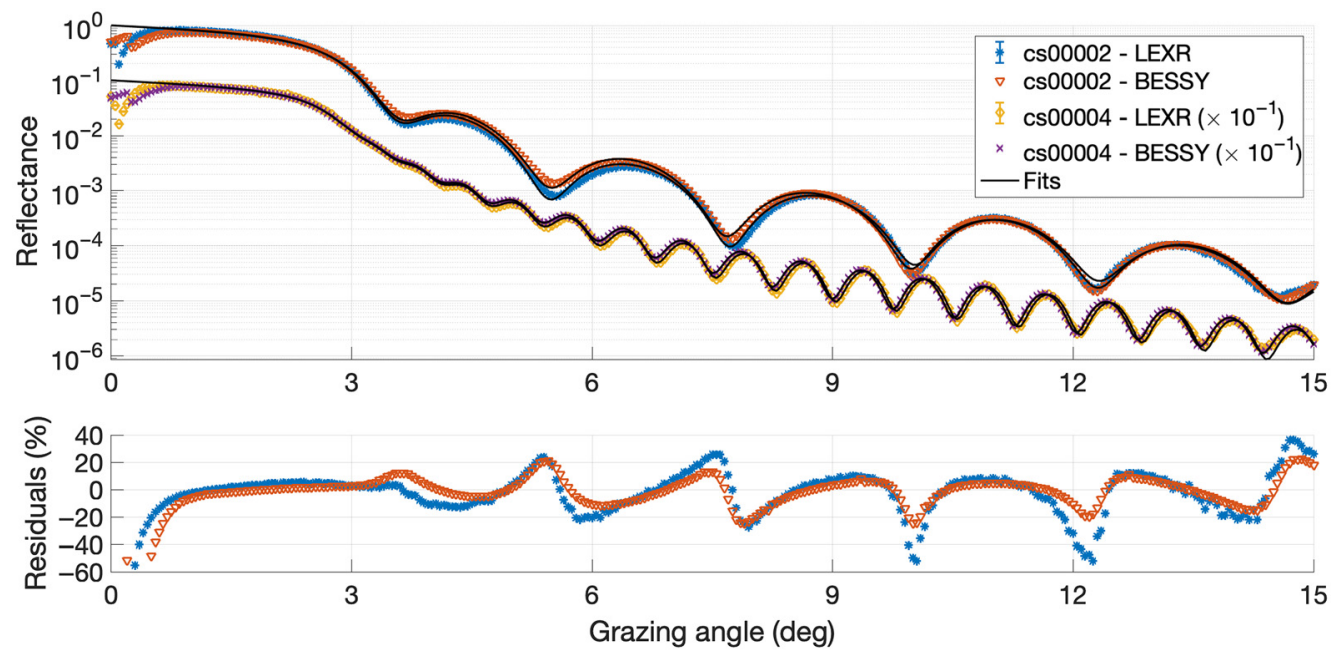

Fig. 21 Specular reflectivity measurements of the same samples using LEXR and BESSY. The lower figure shows the residuals for the fits to cs00002. The fits to cs00004 are off by $< \pm 10 \%$ for the main section of the measurement.

Table 3 Best-fit parameters to 1.487-keV measurements at LEXR and BESSY II. CHO roughness was coupled to that of Ir. The substrate is modeled with a 2-nm overlayer of $\mathrm{SiO}_{2}$ with roughness coupled to that of the Si wafer.

\begin{tabular}{lcccccc}
\hline \hline Sample & Beamline & $\begin{array}{c}\mathrm{CHO} \rho \\
\left(\mathrm{g} / \mathrm{cm}^{3}\right)\end{array}$ & $\begin{array}{c}\mathrm{CHO} z \\
(\mathrm{~nm})\end{array}$ & $\begin{array}{c}\text { Ir z } \\
(\mathrm{nm})\end{array}$ & $\begin{array}{c}\text { Ir } \sigma \\
(\mathrm{nm})\end{array}$ & $\begin{array}{c}\text { Sub } \sigma \\
(\mathrm{nm})\end{array}$ \\
\hline cs00002 & LEXR & 1.3 & 2.0 & 10.0 & 0.25 & 0.37 \\
& BESSY & 0.6 & 1.6 & 10.0 & 0.27 & 0.36 \\
cs00004 & LEXR & 1.4 & 1.7 & 30.7 & 0.30 & 0.39 \\
& BESSY & 1.1 & 1.6 & 30.8 & 0.31 & 0.39 \\
\hline \hline
\end{tabular}

in the fit as lower density and thickness of the hydrocarbon overlayer. The samples were handled multiple times and several months passed between these measurements, which may be the cause of the difference in the contamination layer.

\section{Summary}

We reported on the design, performance, and qualification of ESA's new custom-built low energy x-ray reflectometer to aid in the research and development of thin-film coatings for future $\mathrm{X}$-ray optics. The performance of each subcomponent, as well as the LEXR system as a whole, has been evaluated. In addition to the standard $1.487 \mathrm{keV} \mathrm{Al}$ source, LEXR can be equipped with other sources to facilitate measurements at different energies. In the standard configuration, the reflectometer has a dynamic range of 6 to 8 orders of magnitude depending on measurement conditions, a beam purity of $99.3 \%$ and a divergence of 0.746 arcmin. We have evaluated how the system can be used to probe different characteristic length scales and discussed the bandwidth limits for different measurement techniques. We have discussed measurement repeatability and discussed the effects of hydrocarbons and other surface contaminants on the sample reflectance, and lastly, we have demonstrated agreement between LEXR measurements and measurements performed at the Helmholtz-Zentrum Berlin für Materialien und Energie, BESSY II synchrotron. LEXR is now a part of the suite of characterization tools for ongoing and future ESA projects. The reflectometer has proven to be a useful resource for fast characterization of 
low-density coating behavior and mirror sample performance and is already being utilized to provide valuable data. ${ }^{26,27}$

\section{Acknowledgments}

This activity has been funded by the European Space Agency under contract No. 4000102248/ 10/NL/PM.

\section{References}

1. K. Nandra et al., "The hot and energetic universe: a white paper presenting the science theme motivating the Athena+ mission," astro-ph.HE, arXiv:1306.2307 (2013).

2. A. Abeln et al., "Conceptual design of BabyIAXO, the intermediate stage towards the International Axion Observatory," J. High Energy Phys. 2021(5), 137 (2021).

3. J. A. Gaskin et al., "Lynx X-ray observatory: an overview," J. Astron. Telesc. Instrum. Syst. 5(2), 021001 (2019).

4. S. E. Romaine et al., "Monitoring program for the coating of the AXAF flight optics," Proc. SPIE 2805, 8-17 (1996).

5. R. J. Bruni et al., "Verification of the coating performance for the AXAF flight optics based on reflectivity measurements of coated witness samples," Proc. SPIE 2805, 1-10 (1996).

6. E. Chason and T. M. Mayer, "Thin film and surface characterization by specular x-ray reflectivity," Crit. Rev. Solid State Mater. Sci. 22(1), 1-67 (1997).

7. D. D. M. Ferreira et al., "Design, development, and performance of X-ray mirror coatings for the ATHENA mission," Proc. SPIE 10399, 1039918 (2017).

8. D. D. M. Ferreira et al., "Performance and stability of mirror coatings for the ATHENA mission," Proc. SPIE 10699, 106993K (2018).

9. S. Svendsen et al., "Performance and time stability of Ir/SiC X-ray mirror coatings for ATHENA," Proc. SPIE 11119, $111190 \mathrm{G}$ (2019).

10. S. Massahi et al., "Installation and commissioning of the silicon pore optics coating facility for the ATHENA mission," Proc. SPIE 11119, 111190F (2019).

11. D. Lumb et al., "Influence of a carbon over-coat on the x-ray reflectance of xeus mirrors," Opt. Commun. 279(1), 101-105 (2007).

12. J. Feng et al., "Stability of $\mathrm{cr} / \mathrm{c}$ multilayer during synchrotron radiation exposure and thermal annealing," Opt. Express 27, 38493-38508 (2019).

13. R. F. Elsner, S. L. O'Dell, and M. C. Weisskopf, "Molecular contamination and the calibration of AXAF," Proc. SPIE 1742, 6-12 (1993).

14. S. Achenbach, G. Wells, and C. Shen, "Characterization of the surface contamination of deep x-ray lithography mirrors exposed to synchrotron radiation," J. Synchrotron Radiat. 25, 729-737 (2018).

15. L. Jiang et al., "Investigation of natural contamination layer growth on optical substrates," Chin. Phys. C 42, 115001 (2018).

16. P. L. Henriksen et al., "LEXR: a low-energy x-ray reflectometer for characterization of athena mirror coatings," Proc. SPIE 11119, 111191F (2019).

17. P. L. Henriksen et al., "Qualification and performance of the Low-Energy X-ray Reflectometer (LEXR)," Proc. SPIE 11444, 114444J (2020).

18. F. E. Christensen et al., "Studies of multilayers and thin-foil X-ray mirrors using a soft X-Ray diffractometer," J. X-Ray Sci. Technol. 2, 81-94 (1990).

19. P. Citrin, P. M. Eisenberg, and W. Marra, "Linewidths in X-ray photoemission and X-ray emission spectroscopies: what do they measure?" Phys Rev B 10, 1762 (1974).

20. greateyes GmbH, "Data Sheet: GE-VAC 1024256 Bi DD".

21. W. W. Craig et al., "Fabrication of the NuSTAR flight optics," Proc. SPIE 8147, 81470H (2011).

22. A. Jafari et al., "Long-term performance and durability of Ir/B4C multilayer x-ray mirrors: focusing on composition, structure, and reflectivity properties," J. Astron. Telesc. Instrum. Syst. 6(3), 034005 (2020). 
23. Y. Yang et al., "Comparative study of single-layer, bilayer, and trilayer mirrors with enhanced x-ray reflectance in 0.5- to 8-keV energy region," J. Astron. Telesc. Instrum. Syst. 6(4), 044001 (2020).

24. A. Sokolov et al., "At-wavelength metrology facility for soft x-ray reflection optics," Rev. Sci. Instrum. 87(5), 052005 (2016).

25. S. Massahi et al., "Investigation of boron carbide and iridium thin films, an enabling technology for future x-ray telescopes," Appl. Opt. 59, 10902-10911 (2020).

26. S. Svendsen et al., "Status of the Ir and Ir/SiC coating development for the Athena optics," Proc. SPIE 11444, 114444K (2020).

27. S. Massahi et al., "Balancing of residual stress in thin film iridium by utilizing chromium as an underlayer," Proc. SPIE 11444, 114444N (2020).

Biographies of the authors are not available. 